HETEROCYCLES, Vol. 77, No. 2, 2009, pp. 1105 - 1122. @ The Japan Institute of Heterocyclic Chemistry Received, 31st July, 2008, Accepted, 3rd October, 2008, Published online, 6th October, 2008 DOI: $10.3987 / C O M-08-S(F) 89$

\title{
A GENERAL METHOD FOR THE SYNTHESIS OF $N$-UNSUBSTITUTED 3,4-DIARYLPYRROLE-2,5-DICARBOXYLATES
}

\section{Tsutomu Fukuda, ${ }^{a}$ Yukie Hayashida, ${ }^{a}$ and Masatomo Iwao ${ }^{\text {b* }}$}

${ }^{\mathrm{a}}$ Graduate School of Science and Technology, and ${ }^{\mathrm{b}}$ Department of Applied Chemistry, Faculty of Engineering, Nagasaki University, 1-14 Bunkyo-machi, Nagasaki 852-8521, Japan, e-mail: iwao@nagasaki-u.ac.jp

\begin{abstract}
A general method for the synthesis of $N$-unsubstituted 3,4-diarylpyrrole-2,5-dicarboxylates (3) has been developed. The key reactions involved are the Hinsberg-type synthesis of dimethyl $N$-benzyl-3,4dihydroxypyrrole-2,5-dicarboxylate (6) followed by palladium-catalyzed Suzuki-Miyaura coupling of its bis-triflate derivative (7). The $N$-benzyl protecting group of the resulting 3,4-diarylpyrrole-2,5-dicarboxylates (8) is cleanly removed under hydrogenolytic or solvolytic conditions.
\end{abstract}

\section{INTRODUCTION}

Pyrrole moieties possessing two aryl groups at the 3- and 4-positions appear frequently in marine natural products, such as lamellarins, ${ }_{1}^{1}$ lukianols,${ }^{2}$ ningalins, ${ }^{3}$ storniamides, ${ }^{4}$ polycitones, ${ }^{5}$ purpurone,${ }^{6}$ halitulin, ${ }^{7}$ and dictyodendrins. ${ }^{8}$ These natural products have attracted considerable attention due to their unique structures and highly useful biological activities. As the result, a number of synthetic approaches have been developed so far. ${ }^{1 \mathrm{~b}, 9}$ These syntheses can be divided into two classes depending on the construction method of 3,4-diarylpyrrole scaffold. The first employed de novo ring construction of the pyrrole ring via titanium-mediated reaction, ${ }^{10}$ biomimetic synthesis, ${ }^{11} \mathrm{~N}$-ylide-mediated cyclization, ${ }^{12}$ intra- or intermolecular [3+2] cycloaddition, ${ }^{13,14}$ Diels-Alder cyclization of azadiene, ${ }^{15}$ vinylogous iminium ion-mediated ring formation, ${ }^{16,17}$ and aminoquinone-mediated cyclization. ${ }^{18}$ The second utilized regioselective arylation of preexisting pyrrole core by palladium-catalyzed cross-coupling and related reactions. ${ }^{19}$

In 2003, we reported a short and flexible route to 3,4-diarylpyrrole marine alkaloids. ${ }^{20}$ The key reactions involved are the synthesis of $N$-substituted dimethyl 3,4-dihydroxypyrrole-2,5-dicarboxylates via his $75^{\text {th }}$ birthday. 
Hinsberg-type reaction followed by palladium-catalyzed Suzuki-Miyaura coupling of their bis-triflate derivatives. This strategy has been successfully applied to the total synthesis of biologically significant lamellarins D, L, N and $\alpha 20$-sulfate. ${ }^{21}$ In this paper, we report further application of this strategy for the synthesis of $\mathrm{N}$-unsubstituted dimethyl 3,4-diarylpyrrole-2,5-dicarboxylates.

\section{RESULTS AND DISCUSSION}

Initially, we examined Suzuki-Miyaura coupling of dimethyl 3,4-bis(trifluoromethanesulfonyloxy)pyrrole-2,5-dicarboxylate (1) with 4-methoxyphenylboronic acid (2a). Under the previously established conditions, ${ }^{20}$ bis-triflate (1) was treated with $2 \mathbf{2 a}$ (3.0 equiv.) and aqueous $\mathrm{Na}_{2} \mathrm{CO}_{3}$ in the presence of a catalytic amount of $\mathrm{Pd}\left(\mathrm{PPh}_{3}\right)_{4}$ in refluxing THF for $20 \mathrm{~h}$, however, only trace amount (3\%) of the desired 3,4-bis(4-methoxyphenyl)pyrrole (3a) was obtained and unreacted $\mathbf{1}$ was recovered in $68 \%$ yield (Scheme 1).
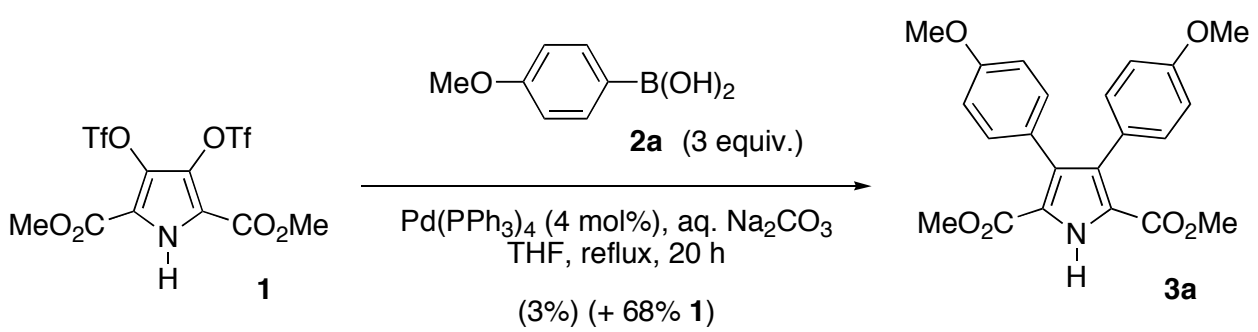

Scheme 1

This result suggested $N$-protection of bis-triflate (1) may be indispensable for the smooth cross-coupling. ${ }^{22}$ Thus, we decided to test the cross-coupling of $N$-benzyl protected bis-triflate (7). The synthesis of $\mathbf{7}$ is shown in Scheme 2. Benzylamine (4) was alkylated with methyl bromoacetate to give $\mathrm{N}$-benzyliminodiacetate (5) in $90 \%$ yield. Hinsberg reaction of $\mathbf{5}$ with dimethyl oxalate using $\mathrm{NaH}$ as a base afforded 3,4-dihydroxypyrrole (6) in $88 \%$ yield. The conventional conditions using NaOMe in $\mathrm{MeOH}$ afforded 6 in much lower yield (58\%). ${ }^{23}$ Triflation of 6 with trifluoromethanesulfonic anhydride in pyridine gave the bis-triflate (7) in $96 \%$ yield.

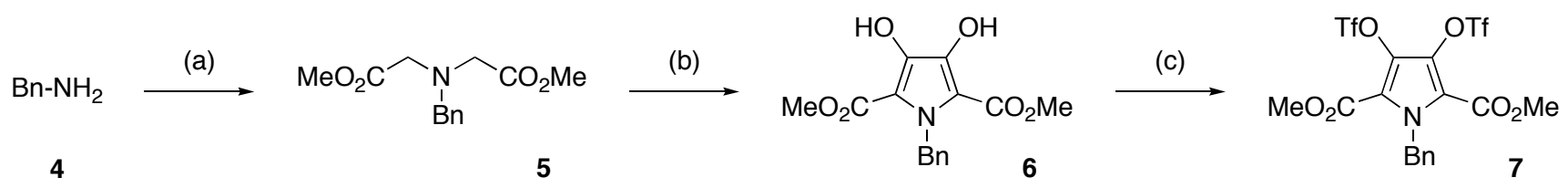

Scheme 2. Reagents and conditions: (a) $\mathrm{BrCH}_{2} \mathrm{CO}_{2} \mathrm{Me}$ (2.1 equiv.), $\mathrm{NaHCO}_{3}, \mathrm{MeCN}$, reflux, $2 \mathrm{~h}$ (90\%). (b) $\left(\mathrm{CO}_{2} \mathrm{Me}\right)_{2}, \mathrm{NaH}$, THF, reflux, $3 \mathrm{~h}(88 \%)$. (c) $\mathrm{Tf}_{2} \mathrm{O}$ (2.2 equiv.), pyridine, $0{ }^{\circ} \mathrm{C}, 1 \mathrm{~h}(96 \%)$.

The results of the cross-coupling of $\mathbf{7}$ with a variety of arylboronic acids (2a-i) are summarized in Table 1. 
In contrast to the reaction of $\mathbf{1 , 7}$ reacted with $\mathbf{2 a - i}$ quite smoothly to give 3,4-diarylated products $\mathbf{8 a - i}$ in excellent yields. The reactions were not affected by electronic or steric effects of the substituents on the aryl ring of the boronic acids.

Table 1. Palladium-catalyzed cross-coupling of bis-triflate (7) with arylboronic acids (2)

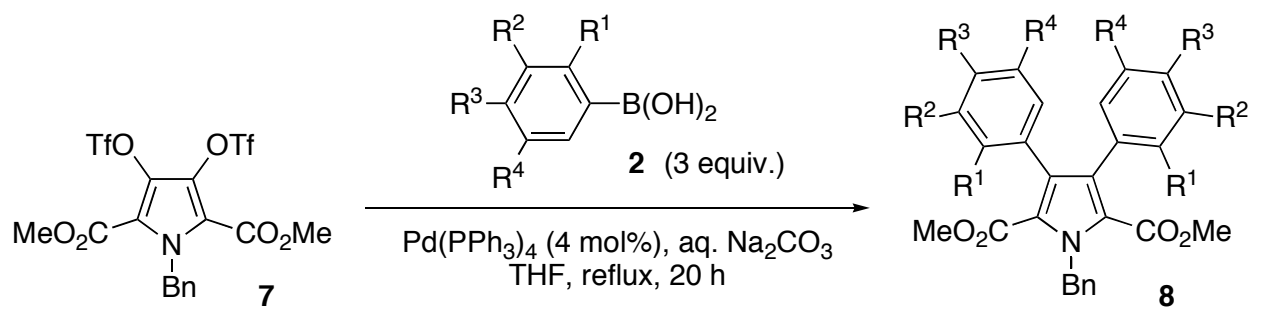

\begin{tabular}{cccccccc}
\hline entry & $\mathbf{2}$ & $\mathrm{R}^{1}$ & $\mathrm{R}^{2}$ & $\mathrm{R}^{3}$ & $\mathrm{R}^{4}$ & $\mathbf{8}$ & ${\text { yield }(\%)^{\mathrm{a}}}^{2}$ \\
\hline 1 & $\mathbf{2 a}$ & $\mathrm{H}$ & $\mathrm{H}$ & $\mathrm{OMe}$ & $\mathrm{H}$ & $\mathbf{8 a}$ & 99 \\
2 & $\mathbf{2 b}$ & $\mathrm{H}$ & $\mathrm{H}$ & $\mathrm{H}$ & $\mathrm{H}$ & $\mathbf{8 b}$ & 98 \\
3 & $\mathbf{2 c}$ & $\mathrm{H}$ & $\mathrm{H}$ & $\mathrm{O} i-\mathrm{Pr}$ & $\mathrm{H}$ & $\mathbf{8 c}$ & quant. \\
4 & $\mathbf{2 d}$ & $\mathrm{H}$ & $\mathrm{H}$ & $\mathrm{F}$ & $\mathrm{H}$ & $\mathbf{8 d}$ & 99 \\
5 & $\mathbf{2 e}$ & $\mathrm{H}$ & $\mathrm{H}$ & $\mathrm{Cl}$ & $\mathrm{H}$ & $\mathbf{8 e}$ & 98 \\
6 & $\mathbf{2 f}$ & $\mathrm{OMe}$ & $\mathrm{H}$ & $\mathrm{H}$ & $\mathrm{H}$ & $\mathbf{8 f}$ & 97 \\
7 & $\mathbf{2 g}$ & $\mathrm{H}$ & $\mathrm{OMe}$ & $\mathrm{OMe}$ & $\mathrm{H}$ & $\mathbf{8 g}$ & 99 \\
8 & $\mathbf{2 h}$ & $\mathrm{H}$ & $\mathrm{OMe}$ & $\mathrm{OMe}$ & $\mathrm{OMe}$ & $\mathbf{8 h}$ & 96 \\
9 & $\mathbf{2 i}$ & $\mathrm{OMOM}$ & $\mathrm{H}$ & $\mathrm{OMe}$ & $\mathrm{OMe}$ & $\mathbf{8 i}$ & 99
\end{tabular}

${ }^{\mathrm{a}}$ Isolated yield.

Having established the cross-coupling reactions of 7, we next carried out debenzylation of 8 to produce $N$-unsubstituted 3,4-diarylpyrroles (3). The results are summarized in Table 2. 3,4-Diarylpyrroles (8a-i) were treated with Pearlman's catalyst ${ }^{24}$ and ammonium formate in refluxing EtOH to give the debenzylated compounds (3) in excellent yields, except for compound (8e). In this case, hydrogenolysis of $\mathrm{C}-\mathrm{Cl}$ bonds on the aryl groups also proceeded and 3,4-diphenylpyrrole (3b) was isolated (entry 5). Fortunately, however, deprotection of $8 \mathbf{e}$ was cleanly effected under solvolytic conditions ${ }^{25}$ (Scheme 3). Since an efficient synthesis of 3,4-symmetrically arylated pyrroles (3a-i) was established, we next examined the stepwise cross-coupling with different arylboronic acids to produce 3,4-unsymmetrically arylated pyrrole (11) (Scheme 4). Thus, the bis-triflate (7) was treated with 1.0 equiv. of 3,4-dimethoxyphenylboronic acid (2g) to give the mono-arylated pyrrole (9) in $75 \%$ yield accompanied by $8 \%$ of $\mathbf{8 g}$. The second cross-coupling of $\mathbf{9}$ with 4-methoxyphenylboronic acid (2a) gave the 3,4-differentially arylated pyrrole (10) in excellent yield. Hydrogenolysis of $\mathbf{1 0}$ gave the deprotected 11 in $69 \%$ yield. 
Table 2. Debenzylation of 3,4-diarylpyrrole (8)
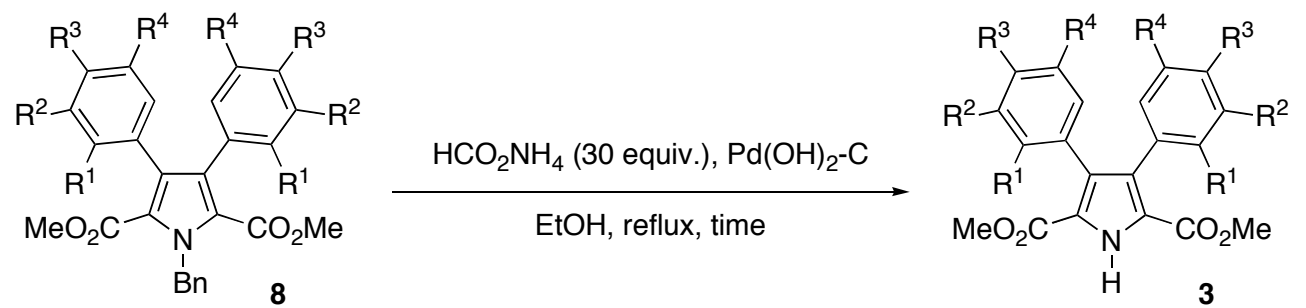

\begin{tabular}{ccccccccc}
\hline entry & $\mathbf{8}$ & $\mathrm{R}^{1}$ & $\mathrm{R}^{2}$ & $\mathrm{R}^{3}$ & $\mathrm{R}^{4}$ & time $(\mathrm{h})$ & $\mathbf{3}$ & ${\text { yield }(\%)^{\mathrm{a}}}^{2}$ \\
\hline 1 & $\mathbf{8 a}$ & $\mathrm{H}$ & $\mathrm{H}$ & $\mathrm{OMe}$ & $\mathrm{H}$ & 1 & $\mathbf{3 a}$ & 97 \\
2 & $\mathbf{8 b}$ & $\mathrm{H}$ & $\mathrm{H}$ & $\mathrm{H}$ & $\mathrm{H}$ & 1 & $\mathbf{3 b}$ & quant. \\
3 & $\mathbf{8 c}$ & $\mathrm{H}$ & $\mathrm{H}$ & $\mathrm{O} i-\mathrm{Pr}$ & $\mathrm{H}$ & 3 & $\mathbf{3 c}$ & 95 \\
4 & $\mathbf{8 d}$ & $\mathrm{H}$ & $\mathrm{H}$ & $\mathrm{F}$ & $\mathrm{H}$ & 3 & $\mathbf{3 d}$ & 89 \\
5 & $\mathbf{8 e}$ & $\mathrm{H}$ & $\mathrm{H}$ & $\mathrm{Cl}$ & $\mathrm{H}$ & 2 & $\mathbf{3 e}$ & $0^{\mathrm{b}}$ \\
6 & $\mathbf{8 f}$ & $\mathrm{OMe}$ & $\mathrm{H}$ & $\mathrm{H}$ & $\mathrm{H}$ & 1 & $\mathbf{3 f}$ & 88 \\
7 & $\mathbf{8 g}$ & $\mathrm{H}$ & $\mathrm{OMe}$ & $\mathrm{OMe}$ & $\mathrm{H}$ & 1.5 & $\mathbf{3 g}$ & 95 \\
8 & $\mathbf{8 h}$ & $\mathrm{H}$ & $\mathrm{OMe}$ & OMe & OMe & 2 & $\mathbf{3 h}$ & 96 \\
9 & $\mathbf{8 i}$ & $\mathrm{OMOM}$ & $\mathrm{H}$ & $\mathrm{OMe}$ & $\mathrm{OMe}$ & 1 & $\mathbf{3 i}$ & 94 \\
\hline
\end{tabular}

${ }^{\mathrm{a}}$ Isolated yield.

${ }^{\mathrm{b}}$ Dimethyl 3,4-diphenylpyrrole-2,5-dicarboxylate (3b) was obtained (85\%).

Finally, we briefly describe the utility of our procedure for the synthesis of natural products. Recently,

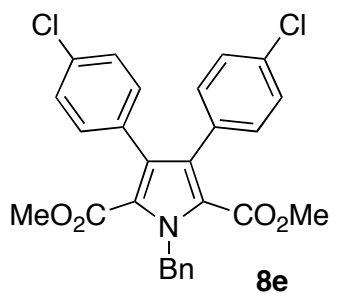

conc. $\mathrm{H}_{2} \mathrm{SO}_{4}$, anisole, TFA

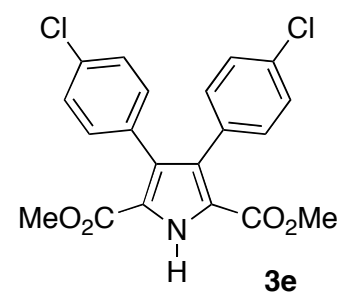

Scheme 3
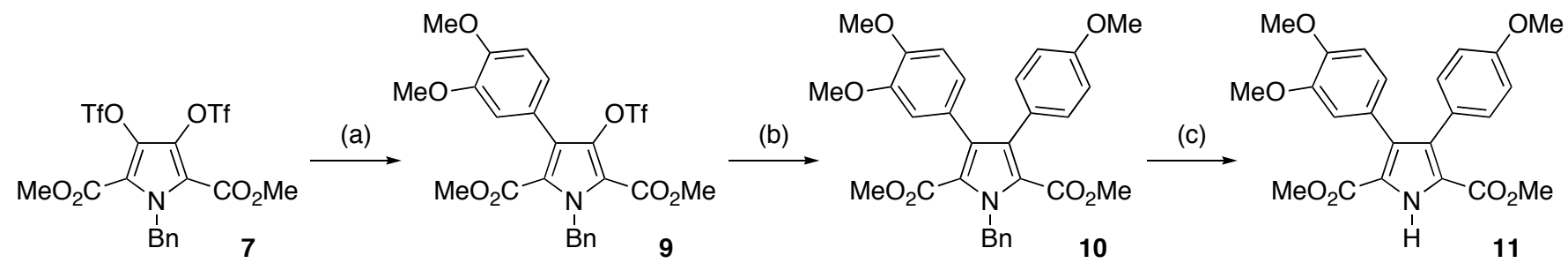

Scheme 4. Reagents and conditions: (a) $2 \mathrm{~g}$ (1 equiv.), $\mathrm{Pd}\left(\mathrm{PPh}_{3}\right)_{4}(2 \mathrm{~mol} \%)$, aq. $\mathrm{Na}_{2} \mathrm{CO}_{3}$, THF, reflux, $4 \mathrm{~h}$ (9: $75 \%, 8 g: 8 \%)$. (b) 2 a (1.5 equiv.), $\mathrm{Pd}\left(\mathrm{PPh}_{3}\right)_{4}(4 \mathrm{~mol} \%)$, aq. $\mathrm{Na}_{2} \mathrm{CO}_{3}, \mathrm{THF}$, reflux, $20 \mathrm{~h}$ (96\%). (c) $\mathrm{HCO}_{2} \mathrm{NH}_{4}$ (30 equiv.), $\mathrm{Pd}(\mathrm{OH})_{2}-\mathrm{C}, \mathrm{EtOH}$, reflux, 3 h (69\%). 
we reported the short-step synthesis of lamellarins $\mathrm{O}, \mathrm{P}, \mathrm{Q}$, and R using $\mathbf{1 3}$ as a common intermediate. ${ }^{26}$ This key intermediate was prepared in two steps from 3c by partial hydrolysis and decarboxylation (Scheme 5).
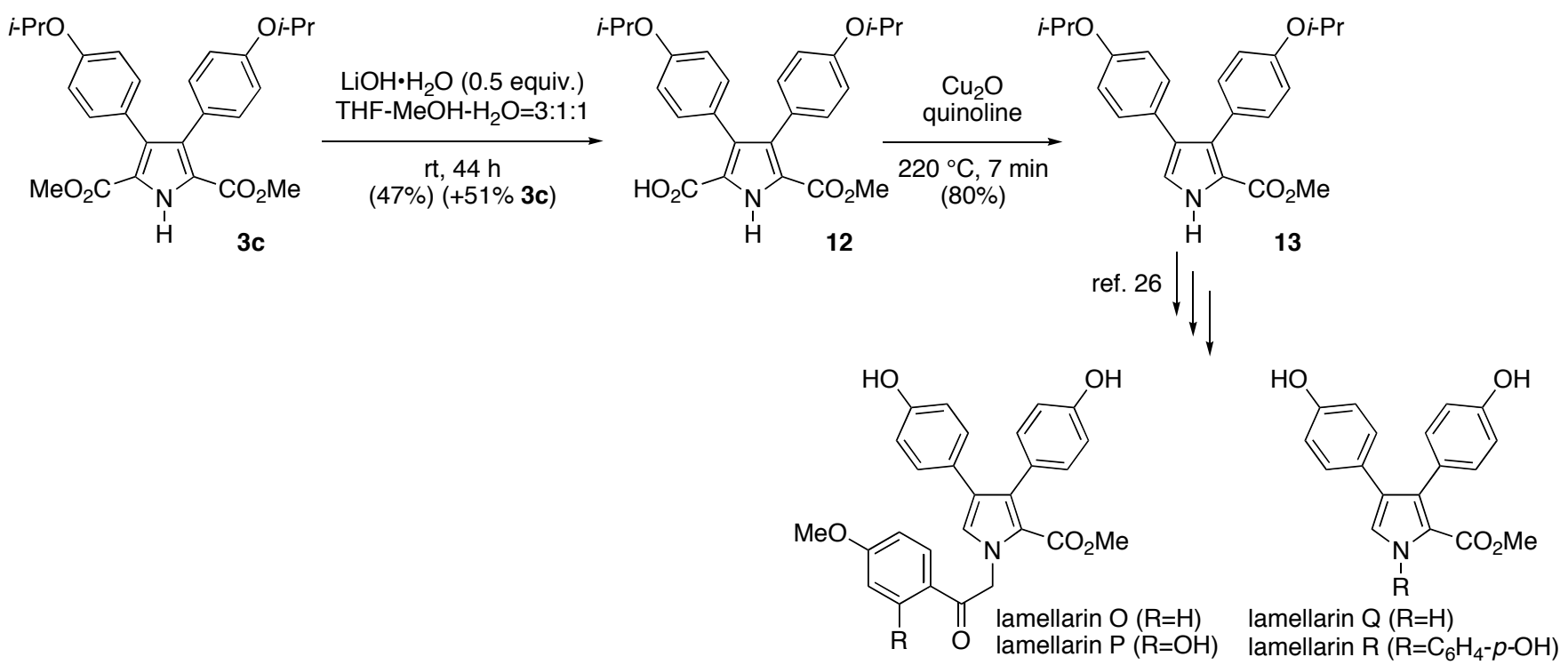

Scheme 5

It is also noteworthy, that the 3,4-diarylpyrroles (3h) and (3i) were utilized as the key intermediates in the synthesis of permethyl storniamide A and ningalin A, respectively, by Boger et al. ${ }^{15 a}$ (Scheme 6).

In conclusion, we have developed an efficient procedure to produce $N$-unsubstituted
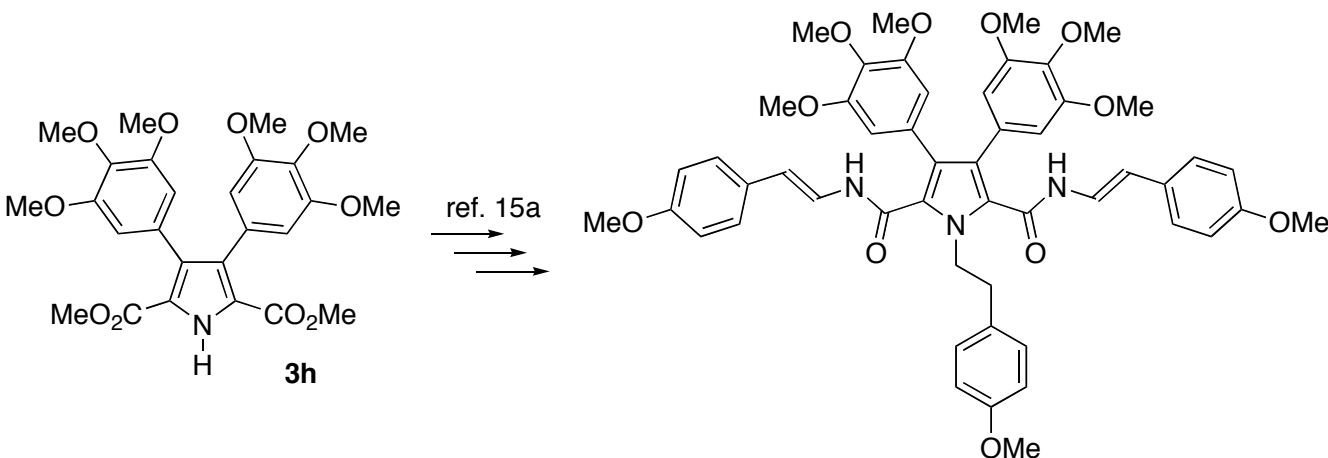

permethyl storniamide A
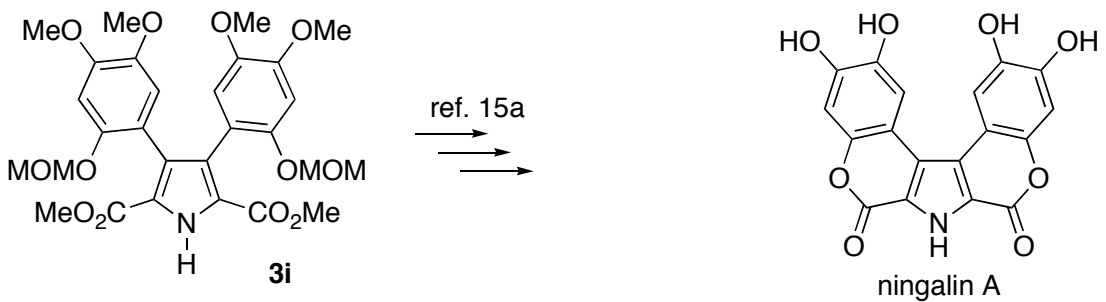

Scheme 6 
3,4-diarylpyrrole-2,5-dicarboxylates. This method can be utilized for the synthesis of 3,4-diarylpyrrole marine natural products and their analogues.

\section{EXPERIMENTAL}

Melting points were determined with a Yanagimoto micro melting points apparatus and are uncorrected. IR spectra were obtained with a Perkin-Elmer System 2000 instrument. NMR spectra were recorded on a JEOL JNM-AL400 instrument (400 MHz for ${ }^{1} \mathrm{H}$ and $100 \mathrm{MHz}$ for ${ }^{13} \mathrm{C}$ ) using tetramethylsilane as an internal standard. High resolution mass spectra were recorded on a JEOL JMS-700N spectrometer. Flash chromatography was conducted on Silica Gel 60N, 40-50 $\mu \mathrm{m}$ (Kanto Chemical Co., Inc.). Column chromatography was conducted on Silica Gel 60N, 63-210 $\mu \mathrm{m}$ (Kanto Chemical Co., Inc.) or Chromatorex NH-DM1020 silica gel (Fuji Silysia Chemical Ltd.). Dry THF were distilled from Na-benzophenone ketyl under argon immediately before use.

\section{Dimethyl 3,4-bis(trifluoromethanesulfonyloxy)pyrrole-2,5-dicarboxylate (1)}

Under an argon atmosphere, a suspension of dimethyl oxalate $(2.36 \mathrm{~g}, 20.0 \mathrm{mmol})$ and $\mathrm{NaH}(60 \%$ dispersion in mineral oil, $1.63 \mathrm{~g}$, ca. $40.8 \mathrm{mmol}$, prewashed with hexane) in THF (10 mL) was heated to reflux. To this suspension was added dropwise a solution of dimethyl iminodiacetate (1.61 g, 10.0 $\mathrm{mmol})$ in THF $(30 \mathrm{~mL})$ under reflux. After being refluxed for additional $3 \mathrm{~h}$, the reaction mixture was cooled to $\mathrm{rt}$, quenched with acetic acid $(3 \mathrm{~mL})$ and evaporated under reduced pressure. The residue was poured into ice-cold water and the suspension was acidified with $2 \mathrm{M}$ aqueous $\mathrm{HCl}$ to $\mathrm{pH} 3$. The precipitated solid was collected by filtration, washed with water, and dried under reduced pressure to give dimethyl 3,4-dihydroxypyrrole-2,5-dicarboxylate as pale yellow powder (1.50 g, 70\%). Recrystallization from $\mathrm{MeOH}$ gave pale yellow powder. $\mathrm{Mp} 180-210^{\circ} \mathrm{C}$ (decomp) (sealed capillary); IR (KBr): 3399, 3302, 1701, 1565, 1500, 1438, 1311, 1194, $1157 \mathrm{~cm}^{-1} ;{ }^{1} \mathrm{H}$ NMR (400 MHz, acetone- $\left.d_{6}\right)$ : $\delta 3.84(\mathrm{~s}, 6 \mathrm{H}), 7.53(\mathrm{~s}, 2 \mathrm{H}), 9.89(\mathrm{~s}, 1 \mathrm{H}) ;{ }^{13} \mathrm{C}$ NMR (100 MHz, acetone- $\left.d_{6}\right): \delta 51.7,109.9,138.5,162.4$. Anal. Calcd for $\mathrm{C}_{8} \mathrm{H}_{9} \mathrm{NO}_{6}$ : C, 44.66; H, 4.22; N, 6.51. Found: C, 44.90; H, 4.33; N, 6.30.

Under an argon atmosphere, trifluoromethanesulfonic anhydride $(3.70 \mathrm{~mL}, 22.6 \mathrm{mmol})$ was added as a neat liquid to a solution of dimethyl 3,4-dihydroxypyrrole-2,5-dicarboxylate (2.19 g, $10.2 \mathrm{mmol})$ in pyridine $(20 \mathrm{~mL})$ at $0{ }^{\circ} \mathrm{C}$. After being stirred for $3 \mathrm{~h}$, the reaction mixture was quenched with water at the same temperature and allowed to warm to rt. The product was extracted with $\mathrm{Et}_{2} \mathrm{O}$ and the extract was washed successively with $3 \mathrm{M}$ aqueous $\mathrm{HCl}$, water, and brine, and dried over $\mathrm{Na}_{2} \mathrm{SO}_{4}$. The solvent was removed by evaporation and the residue was purified by column chromatography over Silica Gel $60 \mathrm{~N}$ (hexane-EtOAc=2:1) to give $\mathbf{1}$ as colorless solid (4.27 g, 87\%). Recrystallization from

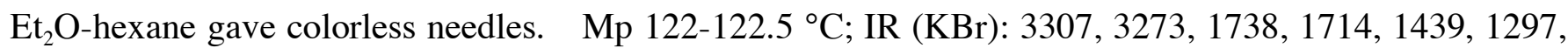


1228, $1135 \mathrm{~cm}^{-1} ;{ }^{1} \mathrm{H}$ NMR (400 MHz, $\mathrm{CDCl}_{3}$ ): $\delta 4.00(\mathrm{~s}, 6 \mathrm{H}), 10.42$ (br s, $\left.1 \mathrm{H}\right) ;{ }^{13} \mathrm{C}$ NMR (100 MHz, $\mathrm{CDCl}_{3}$ ): 52.9, 115.9, $118.4(\mathrm{q}, J=320 \mathrm{~Hz}), 127.4,158.0$. Anal. Calcd for $\mathrm{C}_{10} \mathrm{H}_{7} \mathrm{~F}_{6} \mathrm{NO}_{10} \mathrm{~S}_{2}: \mathrm{C}, 25.06 ; \mathrm{H}$, 1.47; N, 2.92. Found: C, 24.99; H, 1.22; N, 2.87.

\section{Suzuki-Miyaura coupling of bis-triflate (1) with arylboronic acids (2a).}

Under an argon atmosphere, a degassed solution of $\mathrm{Na}_{2} \mathrm{CO}_{3}(700 \mathrm{mg}, 6.60 \mathrm{mmol})$ in water $(2.0 \mathrm{~mL})$ was added to a solution of 1 (479 mg, $1.00 \mathrm{mmol}), \mathbf{2 a}(456 \mathrm{mg}, 3.00 \mathrm{mmol})$ and $\mathrm{Pd}\left(\mathrm{PPh}_{3}\right)_{4}(46.2 \mathrm{mg}, 40.0$ $\mu \mathrm{mol})$ in THF $(20 \mathrm{~mL})$ at $\mathrm{rt}$ and the mixture was refluxed for $20 \mathrm{~h}$. The mixture was cooled to $\mathrm{rt}$ and evaporated under reduced pressure. The residue was diluted with water, adjusted to $\mathrm{pH} 3$ with $1 \mathrm{M}$ aqueous $\mathrm{HCl}$, and saturated with $\mathrm{NaCl}$. The products were extracted with $\mathrm{CH}_{2} \mathrm{Cl}_{2}$ and the extract was washed successively with water and brine, dried over $\mathrm{Na}_{2} \mathrm{SO}_{4}$, and evaporated under reduced pressure. The residue was purified successively by flash chromatography over Silica Gel $60 \mathrm{~N}$ (toluene-EtOAc=5:1 EtOAc) and column chromatography over Chromatorex NH-DM1020 silica gel (hexane-EtOAc=3:1) to give 3a as colorless solid (12.0 mg, 3\%) and unreacted $\mathbf{1}(326 \mathrm{mg}, 68 \%)$.

\section{Dimethyl $N$-benzyliminodiacetate (5)}

Methyl bromoacetate $(90 \mathrm{~mL}, 0.951 \mathrm{~mol}$ ) was added as a neat liquid to a suspension of benzylamine (4) $(49 \mathrm{~mL}, 0.449 \mathrm{~mol})$ and $\mathrm{NaHCO}_{3}(159 \mathrm{~g}, 1.89 \mathrm{~mol})$ in $\mathrm{MeCN}(700 \mathrm{~mL})$ at $\mathrm{rt}$. The mixture was refluxed for $2 \mathrm{~h}$ and then cooled to rt. After removal of inorganic salts by filtration, the filtrate was evaporated under reduced pressure. The residue was purified by distillation $\left(112^{\circ} \mathrm{C} / 0.2 \mathrm{mmHg}\right)$ to give 5 as pale yellow oil (101 g, 90\%). IR (neat): 1746, 1454, 1436, 1202, $1010 \mathrm{~cm}^{-1} ;{ }^{1} \mathrm{H}$ NMR (400 MHz, $\left.\mathrm{CDCl}_{3}\right): \delta$ $3.56(\mathrm{~s}, 4 \mathrm{H}), 3.70(\mathrm{~s}, 6 \mathrm{H}), 3.91(\mathrm{~s}, 2 \mathrm{H}), 7.23-7.29(\mathrm{~m}, 1 \mathrm{H}), 7.29-7.35(\mathrm{~m}, 2 \mathrm{H}), 7.35-7.40(\mathrm{~m}, 2 \mathrm{H}) ;{ }^{13} \mathrm{C}$ NMR (100 MHz, $\left.\mathrm{CDCl}_{3}\right): \delta 51.5,54.0,58.0,127.4,128.4,129.0,138.1,171.6 . \quad$ HREIMS $m / z$. Calcd for $\mathrm{C}_{13} \mathrm{H}_{17} \mathrm{NO}_{4}\left(\mathrm{M}^{+}\right): 251.1158$. Found: 251.1155.

\section{Dimethyl $N$-benzyl-3,4-dihydroxypyrrole-2,5-dicarboxylate (6)}

Under an argon atmosphere, a suspension of dimethyl oxalate (14.9 g, $126 \mathrm{mmol})$ and $\mathrm{NaH}(60 \%$ dispersion in mineral oil, $10.0 \mathrm{~g}$, ca. $250 \mathrm{mmol}$, prewashed with hexane) in THF (80 mL) was heated to reflux. To this suspension was added dropwise a solution of 5 (15.7 g, $62.5 \mathrm{mmol})$ in THF $(150 \mathrm{~mL})$ under reflux. After being refluxed for additional $3 \mathrm{~h}$, the reaction mixture was cooled to $\mathrm{rt}$, quenched with acetic acid $(16 \mathrm{~mL})$ and evaporated under reduced pressure. The residue was poured into ice-cold water and the suspension was acidified with $2 \mathrm{M}$ aqueous $\mathrm{HCl}$ to $\mathrm{pH} 3$. The precipitated solid was collected by filtration, washed with water, and dried under reduced pressure to give 6 as pale brown powder $(16.8 \mathrm{~g}, 88 \%)$. Recrystallization from $\mathrm{MeOH}$ gave pale brown powder. $\mathrm{Mp} 166.5-167{ }^{\circ} \mathrm{C}$; IR 
(KBr): 3365, 1692, 1658, 1509, 1461, 1296, 1195, $1160 \mathrm{~cm}^{-1} ;{ }^{1} \mathrm{H}$ NMR (400 MHz, $\left.\mathrm{CDCl}_{3}\right): \delta 3.85$ (s, 6H), $5.75(\mathrm{~s}, 2 \mathrm{H}), 6.90-6.94(\mathrm{~m}, 2 \mathrm{H}), 7.16-7.20(\mathrm{~m}, 3 \mathrm{H}), 7.65(\mathrm{br} \mathrm{s}, 2 \mathrm{H}) ;{ }^{13} \mathrm{C} \mathrm{NMR}\left(100 \mathrm{MHz}, \mathrm{CDCl}_{3}\right): \delta 49.3$, 51.8, 111.0, 125.6, 126.9, 128.4, 138.9, 139.4, 162.5. Anal. Calcd for $\mathrm{C}_{15} \mathrm{H}_{15} \mathrm{NO}_{6}$ : C, 59.01; H, 4.95; N, 4.59. Found: C, 59.07; H, 4.85; N, 4.55.

\section{Dimethyl $N$-benzyl-3,4-bis(trifluoromethanesulfonyloxy)pyrrole-2,5-dicarboxylate (7)}

Under an argon atmosphere, trifluoromethanesulfonic anhydride $(18.2 \mathrm{~mL}, 108 \mathrm{mmol})$ was added as a neat liquid to a solution of $6(15.0 \mathrm{~g}, 49.1 \mathrm{mmol})$ in pyridine $(75 \mathrm{~mL})$ at $0{ }^{\circ} \mathrm{C}$. After being stirred for $1 \mathrm{~h}$, the reaction mixture was quenched with water at the same temperature and allowed to warm to rt. The product was extracted with $\mathrm{Et}_{2} \mathrm{O}$ and the extract was washed successively with $3 \mathrm{M}$ aqueous $\mathrm{HCl}$, water, and brine, and dried over $\mathrm{Na}_{2} \mathrm{SO}_{4}$. The solvent was removed by evaporation and the residue was purified by column chromatography over Silica Gel 60N (hexane-EtOAc=5:1) to give 7 as colorless solid $(26.9 \mathrm{~g}, 96 \%)$. Recrystallization from $\mathrm{Et}_{2} \mathrm{O}$-hexane gave colorless granules. $\mathrm{Mp} 70.5-71{ }^{\circ} \mathrm{C}$; $\mathrm{IR}(\mathrm{KBr})$ : 1745, 1438, 1289, 1248, 1211, $1134 \mathrm{~cm}^{-1} ;{ }^{1} \mathrm{H}$ NMR (400 MHz, $\left.\mathrm{CDCl}_{3}\right): \delta 3.88(\mathrm{~s}, 6 \mathrm{H}), 6.19(\mathrm{~s}, 2 \mathrm{H})$, 6.95-7.02 (m, 2H), 7.21-7.34 (m, 3H); $\left.{ }^{13} \mathrm{C} \mathrm{NMR} \mathrm{(100} \mathrm{MHz,} \mathrm{CDCl}_{3}\right): \delta 49.8,52.4,117.6,118.4$ (q, $J=320$ $\mathrm{Hz})$, 126.0, 127.6, 128.3, 128.7, 136.2, 158.0. Anal. Calcd for $\mathrm{C}_{17} \mathrm{H}_{13} \mathrm{~F}_{6} \mathrm{NO}_{10} \mathrm{~S}_{2}: \mathrm{C}, 35.86 ; \mathrm{H}, 2.30 ; \mathrm{N}$, 2.46. Found: C, 35.64; H, 2.12; N, 2.42 .

\section{Suzuki-Miyaura coupling of bis-triflate (7) with arylboronic acids (2). General procedure}

Under an argon atmosphere, a degassed solution of $\mathrm{Na}_{2} \mathrm{CO}_{3}(2.10 \mathrm{~g}, 19.8 \mathrm{mmol})$ in water $(6.0 \mathrm{~mL})$ was added to a solution of $7(3.02 \mathrm{mmol}), 2(9.02 \mathrm{mmol})$ and $\mathrm{Pd}\left(\mathrm{PPh}_{3}\right)_{4}(140 \mathrm{mg}, 0.121 \mathrm{mmol})$ in THF (60 $\mathrm{mL}$ ) at $\mathrm{rt}$ and the mixture was refluxed for $20 \mathrm{~h}$. The mixture was cooled to $\mathrm{rt}$ and evaporated under reduced pressure. The product was extracted with $\mathrm{CH}_{2} \mathrm{Cl}_{2}$ and the extract was washed successively with water and brine, dried over $\mathrm{Na}_{2} \mathrm{SO}_{4}$, and evaporated under reduced pressure. The residue was purified by column chromatography to give $\mathbf{8}$.

\section{Dimethyl $N$-benzyl-3,4-bis(4-methoxyphenyl)pyrrole-2,5-dicarboxylate (8a)}

According to the general procedure, $7(1.72 \mathrm{~g}, 3.02 \mathrm{mmol})$ and $2 \mathrm{a}(1.37 \mathrm{~g}, 9.02 \mathrm{mmol})$ were reacted. After chromatographic purification over Silica Gel 60N (toluene toluene-EtOAc=10:1), 8a was obtained as colorless solid $(1.45 \mathrm{~g}, 99 \%)$. Recrystallization from $\mathrm{CH}_{2} \mathrm{Cl}_{2}$-hexane gave colorless granules. $\mathrm{Mp}$ 142-143 ${ }^{\circ} \mathrm{C}$; IR (KBr): 1717, 1694, 1534, 1431, 1293, 1246, 1203, $1032 \mathrm{~cm}^{-1}$; ${ }^{1} \mathrm{H}$ NMR (400 MHz, $\left.\mathrm{CDCl}_{3}\right): \delta 3.52(\mathrm{~s}, 6 \mathrm{H}), 3.75(\mathrm{~s}, 6 \mathrm{H}), 6.01(\mathrm{~s}, 2 \mathrm{H}), 6.69-6.73(\mathrm{~m}, 4 \mathrm{H}), 6.93-6.96(\mathrm{~m}, 4 \mathrm{H}), 7.07-7.11(\mathrm{~m}$, 2H), 7.18-7.24 (m, 1H), 7.26-7.32 (m, 2H); $\left.{ }^{13} \mathrm{C} \mathrm{NMR} \mathrm{(100} \mathrm{MHz,} \mathrm{CDCl}_{3}\right): \delta 49.7,51.4,55.1,112.7,124.4$, 126.2, 126.4, 126.9, 128.4, 130.9, 131.4, 138.6, 158.1, 162.0. Anal. Calcd for $\mathrm{C}_{29} \mathrm{H}_{27} \mathrm{NO}_{6}: \mathrm{C}, 71.74 ; \mathrm{H}$, 
5.61; N, 2.88. Found: C, 71.52; H, 5.62; N, 2.84.

\section{Dimethyl $N$-benzyl-3,4-diphenylpyrrole-2,5-dicarboxylate $(8 b)$}

According to the general procedure, $7(1.71 \mathrm{~g}, 3.00 \mathrm{mmol})$ and $\mathbf{2 b}(1.10 \mathrm{~g}, 9.00 \mathrm{mmol})$ were reacted. After chromatographic purification over Silica Gel 60N (toluene), 8b was obtained as colorless solid $(1.25 \mathrm{~g}, 98 \%)$. Recrystallization from $\mathrm{Et}_{2} \mathrm{O}$-pentane gave colorless needles. $\quad \mathrm{Mp} 138-139{ }^{\circ} \mathrm{C}$; IR $(\mathrm{KBr})$ : 1716, 1435, 1296, 1228, 1201, $1173 \mathrm{~cm}^{-1} ;{ }^{1} \mathrm{H}$ NMR (400 MHz, $\mathrm{CDCl}_{3}$ ): $\delta 3.49$ (s, 6H), 6.05 (s, 2H), 7.01-7.06 (m, 4H), 7.09-7.25 (m, 9H), 7.27-7.33 (m, 2H); $\left.{ }^{13} \mathrm{C} \mathrm{NMR} \mathrm{(100} \mathrm{MHz,} \mathrm{CDCl}_{3}\right): \delta 49.6,51.4$, 124.4 , 126.2 126.4, 126.9, 127.1, 128.3, 130.2, 131.1, 134.0, 138.4, 161.8. Anal. Calcd for $\mathrm{C}_{27} \mathrm{H}_{23} \mathrm{NO}_{4}$ : C, 76.22; H, 5.45; N, 3.29. Found: C, 76.05; H, 5.38; N, 3.22.

\section{Dimethyl $N$-benzyl-3,4-bis(4-isopropoxyphenyl)pyrrole-2,5-dicarboxylate (8c)}

According to the general procedure, $7(5.29 \mathrm{~g}, 9.29 \mathrm{mmol})$ and $2 \mathrm{c}(5.02 \mathrm{~g}, 27.9 \mathrm{mmol})$ were reacted. After chromatographic purification over Silica Gel 60N (toluene toluene-EtOAc=10:1), 8c was obtained as colorless solid (5.02 g, quant.). Recrystallization from $\mathrm{Et}_{2} \mathrm{O}$-pentane gave colorless. $\mathrm{Mp}$ 140-141 ${ }^{\circ} \mathrm{C}$; IR (KBr): 1713, 1531, 1435, 1298, 1244, 1201, $1182 \mathrm{~cm}^{-1} ;{ }^{1} \mathrm{H}$ NMR (400 MHz, $\left.\mathrm{CDCl}_{3}\right): \delta$ $1.30(\mathrm{~d}, J=6.1 \mathrm{~Hz}, 12 \mathrm{H}), 3.52(\mathrm{~s}, 6 \mathrm{H}), 4.43-4.53(\mathrm{~m}, 2 \mathrm{H}), 6.00(\mathrm{~s}, 2 \mathrm{H}), 6.68-6.72(\mathrm{~m}, 4 \mathrm{H}), 6.90-6.94(\mathrm{~m}$, 4H), 7.07-7.10 (m, 2H), 7.18-7.23 (m, 1H), 7.26-7.31 (m, 2H); $\left.{ }^{13} \mathrm{C} \mathrm{NMR} \mathrm{(100} \mathrm{MHz,} \mathrm{CDCl}_{3}\right): \delta 22.0,49.6$, 51.3, 69.7, 114.7, 124.3, 126.2, 126.2, 126.9, 128.3, 130.8, 131.4, 138.6, 156.3, 162.0. Anal. Calcd for $\mathrm{C}_{33} \mathrm{H}_{35} \mathrm{NO}_{6}: \mathrm{C}, 73.18 ; \mathrm{H}, 6.51 ; \mathrm{N}, 2.59$. Found: C, 73.31; H, 6.63; N, 2.47.

\section{Dimethyl $N$-benzyl-3,4-bis(4-fluorophenyl)pyrrole-2,5-dicarboxylate (8d)}

According to the general procedure, $7(1.71 \mathrm{~g}, 3.00 \mathrm{mmol})$ and $\mathbf{2 d}(1.26 \mathrm{~g}, 9.01 \mathrm{mmol})$ were reacted. After chromatographic purification over Silica Gel 60N (toluene), 8d was obtained as colorless solid $(1.37 \mathrm{~g}, 99 \%)$. Recrystallization from $\mathrm{CH}_{2} \mathrm{Cl}_{2}$-hexane gave colorless granules. Mp 126.5-127.5 ${ }^{\circ} \mathrm{C}$; IR (KBr): 1714, 1534, 1436, 1299, 1225, 1201, $1176 \mathrm{~cm}^{-1} ;{ }^{1} \mathrm{H}$ NMR (400 MHz, $\left.\mathrm{CDCl}_{3}\right): \delta 3.52(\mathrm{~s}, 6 \mathrm{H}), 6.05$ (s, 2H), 6.84-6.91 (m, 4H), 6.95-7.01 (m, 4H), 7.08-7.11 (m, 2H), 7.20-7.26 (m, 1H), 7.27-7.33 (m, 2H); ${ }^{13} \mathrm{C}$ NMR (100 MHz, $\left.\mathrm{CDCl}_{3}\right): \delta 49.8,51.5,114.3(\mathrm{~d}, J=21 \mathrm{~Hz}), 124.5,126.3,127.1,128.5,129.9$ (d, $J=$ $3.3 \mathrm{~Hz}), 130.3,131.9(\mathrm{~d}, J=8.3 \mathrm{~Hz}), 138.4,161.6(\mathrm{~d}, J=245 \mathrm{~Hz}), 161.6$. Anal. Calcd for $\mathrm{C}_{27} \mathrm{H}_{21} \mathrm{~F}_{2} \mathrm{NO}_{4}$ : C, 70.28; H, 4.59; N, 3.04. Found: C, 70.56; H, 4.61; N, 2.98 .

\section{Dimethyl $N$-benzyl-3,4-bis(4-chlorophenyl)pyrrole-2,5-dicarboxylate (8e)}

According to the general procedure, $7(1.71 \mathrm{~g}, 3.00 \mathrm{mmol})$ and $2 \mathrm{e}(1.41 \mathrm{~g}, 9.02 \mathrm{mmol})$ were reacted. After chromatographic purification over Silica Gel 60N (toluene), 8e was obtained as colorless solid (1.45 
g, 98\%). Recrystallization from $\mathrm{CH}_{2} \mathrm{Cl}_{2}$-hexane gave colorless prisms. Mp 128-129 ${ }^{\circ} \mathrm{C}$; $\mathrm{IR}(\mathrm{KBr})$ : 1707, 1455, 1434, 1292, 1196, 1171, 1102, $1020 \mathrm{~cm}^{-1} ;{ }^{1} \mathrm{H}$ NMR (400 MHz, $\left.\mathrm{CDCl}_{3}\right): \delta 3.52$ (s, 6H), 6.05 (s, 2H), 6.93-6.96 (m, 4H), 7.07-7.11 (m, 2H), 7.14-7.18 (m, 4H), 7.20-7.25 (m, 1H), 7.27-7.33 (m, 2H);

${ }^{13} \mathrm{C}$ NMR $\left(100 \mathrm{MHz}, \mathrm{CDCl}_{3}\right): \delta 49.8,51.6,124.5,126.3,127.1,127.6,128.5,129.9,131.6,132.4,132.7$, 138.2, 161.5. Anal. Calcd for $\mathrm{C}_{27} \mathrm{H}_{21} \mathrm{Cl}_{2} \mathrm{NO}_{4}: \mathrm{C}, 65.60 ; \mathrm{H}, 4.28 ; \mathrm{N}, 2.83$. Found: C, 65.54; $\mathrm{H}, 4.27 ; \mathrm{N}$, 2.72 .

\section{Dimethyl $N$-benzyl-3,4-bis(2-methoxyphenyl)pyrrole-2,5-dicarboxylate (8f)}

According to the general procedure, 7 (564 mg, $0.990 \mathrm{mmol})$ and $2 \mathbf{f}(453 \mathrm{mg}, 2.98 \mathrm{mmol})$ were reacted. After successive purification by column chromatography over Silica Gel $60 \mathrm{~N}$ (toluene-EtOAc=20:1 10:1) and column chromatography over Silica Gel $60 \mathrm{~N}$ (hexane- $\mathrm{CH}_{2} \mathrm{Cl}_{2}=1: 5$ ), 8f was obtained as colorless solid (466 mg, 97\%). Recrystallization from $\mathrm{CH}_{2} \mathrm{Cl}_{2}-\mathrm{Et}_{2} \mathrm{O}$ gave colorless granules. $\quad$ Mp 168.5-169.5 ${ }^{\circ} \mathrm{C}$; IR (KBr): 1721, 1698, 1438, 1291, 1248, 1201, 1176, $1029 \mathrm{~cm}^{-1} ;{ }^{1} \mathrm{H}$ NMR (400 MHz, $\left.\mathrm{CDCl}_{3}\right): \delta 3.47(\mathrm{~s}, 6 \mathrm{H}), 3.57(\mathrm{~s}, 6 \mathrm{H}), 6.10(\mathrm{~s}, 2 \mathrm{H}), 6.69-6.77(\mathrm{~m}, 4 \mathrm{H}), 6.86-6.90(\mathrm{~m}, 2 \mathrm{H})$, 7.09-7.15 (m, 4H), 7.16-7.22 (m, 1H), 7.25-7.31 (m, 2H); $\left.{ }^{13} \mathrm{C} \mathrm{NMR} \mathrm{(100} \mathrm{MHz,} \mathrm{CDCl}_{3}\right): \delta 49.6,51.2,55.3$, 110.0, 119.6, 124.0, 125.1, 126.2, 126.7, 127.2, 127.9, 128.3, 131.3, 138.9, 156.8, 161.9. Anal. Calcd for $\mathrm{C}_{29} \mathrm{H}_{27} \mathrm{NO}_{6}$ : C, 71.74; H, 5.61; N, 2.88. Found: C, 71.62; H, 5.71; N, 2.72.

\section{Dimethyl $N$-benzyl-3,4-bis(3,4-dimethoxyphenyl)pyrrole-2,5-dicarboxylate $(8 \mathrm{~g})$}

According to the general procedure, $7(1.71 \mathrm{~g}, 3.00 \mathrm{mmol})$ and $2 \mathrm{~g}(1.64 \mathrm{~g}, 9.01 \mathrm{mmol})$ were reacted. After chromatographic purification over Silica Gel 60N (toluene-EtOAc=5:1), 8g was obtained as colorless solid (1.62 g, 99\%). Recrystallization from $\mathrm{CH}_{2} \mathrm{Cl}_{2}$-hexane gave colorless needles. $\mathrm{Mp}$ 154.5-155.5 ${ }^{\circ} \mathrm{C}$; IR (KBr): 1718, 1532, 1439, 1318, 1235, 1206, 1178, $1028 \mathrm{~cm}^{-1}$; ${ }^{1} \mathrm{H}$ NMR (400 MHz, $\left.\mathrm{CDCl}_{3}\right): \delta 3.56(\mathrm{~s}, 6 \mathrm{H}), 3.65(\mathrm{~s}, 6 \mathrm{H}), 3.84(\mathrm{~s}, 6 \mathrm{H}), 6.00(\mathrm{~s}, 2 \mathrm{H}), 6.55(\mathrm{~d}, J=1.7 \mathrm{~Hz}, 2 \mathrm{H}), 6.61(\mathrm{dd}, J=1.7$ and $8.1 \mathrm{~Hz}, 2 \mathrm{H}), 6.71(\mathrm{~d}, J=8.1 \mathrm{~Hz}, 2 \mathrm{H}), 7.11-7.14(\mathrm{~m}, 2 \mathrm{H}), 7.20-7.26(\mathrm{~m}, 1 \mathrm{H}), 7.28-7.34(\mathrm{~m}, 2 \mathrm{H}) ;{ }^{13} \mathrm{C}$ NMR (100 MHz, $\left.\mathrm{CDCl}_{3}\right): \delta 49.6,51.5,55.7,110.1,113.8,122.7,124.3,126.3,126.6,127.0,128.4,130.6$, 138.4, 147.5, 147.7, 162.0. Anal. Calcd for $\mathrm{C}_{31} \mathrm{H}_{31} \mathrm{NO}_{8}: \mathrm{C}, 68.25 ; \mathrm{H}, 5.73 ; \mathrm{N}, 2.57$. Found: C, 68.09; $\mathrm{H}$, $5.86 ; \mathrm{N}, 2.51$.

\section{Dimethyl $N$-benzyl-3,4-bis(3,4,5-trimethoxyphenyl)pyrrole-2,5-dicarboxylate $(8 \mathrm{~h})$}

According to the general procedure, $7(410 \mathrm{mg}, 0.720 \mathrm{mmol})$ and $\mathbf{2 h}(455 \mathrm{mg}, 2.15 \mathrm{mmol})$ were reacted. After successive purification by column chromatography over Silica Gel 60N (toluene-EtOAc=10:1 5:1) and column chromatography over Chromatorex NH-DM1020 silica gel (toluene-EtOAc=10:1), 8h was obtained as colorless solid (418 mg, 96\%). Recrystallization from $\mathrm{CH}_{2} \mathrm{Cl}_{2}-\mathrm{Et}_{2} \mathrm{O}$ gave colorless granules. 
Mp 159.5-160.5 ${ }^{\circ} \mathrm{C}$; IR (KBr): 1717, 1582, 1434, 1341, 1279, 1239, 1196, 1128, 1099, $1001 \mathrm{~cm}^{-1} ;{ }^{1} \mathrm{H}$ NMR (400 MHz, $\left.\mathrm{CDCl}_{3}\right): \delta 3.59(\mathrm{~s}, 6 \mathrm{H}), 3.64(\mathrm{~s}, 12 \mathrm{H}), 3.81(\mathrm{~s}, 6 \mathrm{H}), 5.99(\mathrm{~s}, 2 \mathrm{H}), 6.28(\mathrm{~s}, 4 \mathrm{H}), 7.13-7.18$ $(\mathrm{m}, 2 \mathrm{H}), 7.22-7.24(\mathrm{~m}, 1 \mathrm{H}), 7.27-7.35(\mathrm{~m}, 2 \mathrm{H}) ;{ }^{13} \mathrm{C} \mathrm{NMR}\left(100 \mathrm{MHz}, \mathrm{CDCl}_{3}\right): \delta 49.7,51.7,56.1,60.9$, 107.9, 124.2, 126.4, 127.1, 128.4, 129.4, 130.4, 136.8, 138.2, 152.2, 161.9. Anal. Calcd for $\mathrm{C}_{33} \mathrm{H}_{35} \mathrm{NO}_{10}$ : C, 65.44; H, 5.82; N, 2.31. Found: C, 65.73; H, 5.87; N, 2.19.

\section{Dimethyl $N$-benzyl-3,4-bis(4,5-dimethoxy-2-methoxymethoxyphenyl)pyrrole-2,5-dicarboxylate (8i)} According to the general procedure, $7(2.29 \mathrm{~g}, 4.02 \mathrm{mmol})$ and $2 \mathbf{i}(2.90 \mathrm{~g}, 12.0 \mathrm{mmol})$ were reacted. After successive purification by column chromatography over Silica Gel 60N (hexane-EtOAc=1:1) and column chromatography over Chromatorex NH-DM1020 silica gel (hexane-EtOAc=2:1), 8i was obtained as colorless solid (2.65 g, 99\%). Recrystallization from $\mathrm{CH}_{2} \mathrm{Cl}_{2}-\mathrm{Et}_{2} \mathrm{O}$ gave colorless granules. $\mathrm{Mp}$ 139.5-140.5 ${ }^{\circ} \mathrm{C}$; IR (KBr): 1722, 1703, 1533, 1439, 1288, 1214, 1078, 1031, $1005 \mathrm{~cm}^{-1}$; ${ }^{1} \mathrm{H}$ NMR (400 $\mathrm{MHz} \mathrm{CDCl}_{3}$ ): $\delta 3.28(\mathrm{~s}, 6 \mathrm{H}), 3.55(\mathrm{~s}, 6 \mathrm{H}), 3.56(\mathrm{~s}, 6 \mathrm{H}), 3.83(\mathrm{~s}, 6 \mathrm{H}), 4.83(\mathrm{br} \mathrm{s}, 4 \mathrm{H}), 6.06(\mathrm{~s}, 2 \mathrm{H}), 6.47$ (br s, 2H), 6.68 (s, 2H), 7.12-7.16 (m, 2H), 7.19-7.24 (m, 1H), 7.26-7.33 (m, 2H); ${ }^{13} \mathrm{C} \mathrm{NMR} \mathrm{(100} \mathrm{MHz,}$ $\left.\mathrm{CDCl}_{3}\right): \delta 49.5,51.5,55.8,55.9,56.0,96.6,101.7,114.4,117.1,125.3,126.5,126.8,127.0,128.4,138.9$, 143.7, 148.5, 149.2, 162.2. Anal. Calcd for $\mathrm{C}_{35} \mathrm{H}_{39} \mathrm{NO}_{12}: \mathrm{C}, 63.15 ; \mathrm{H}, 5.91 ; \mathrm{N}, 2.10$. Found: C, 63.12; H, 5.97; N, 1.99 .

\section{Synthesis of dimethyl 3,4-diarylpyrrole-2,5-dicarboxylates (3). General procedure}

Under an argon atmosphere, ammonium formate $(1.53 \mathrm{~g}, 24.3 \mathrm{mmol})$ was added portionwise to a mixture of $8(0.801 \mathrm{mmol})$, Pearlman's catalyst $(129 \mathrm{mg})$, and EtOH $(16 \mathrm{~mL})$ at rt. After being refluxed for an appropriate reaction time shown in Table 2, the mixture was cooled to $\mathrm{rt}$ and passed through a pad of Celite. The filtrate was evaporated under reduced pressure. The residue was purified by column chromatography to give 3 .

\section{Dimethyl 3,4-bis(4-methoxyphenyl)pyrrole-2,5-dicarboxylate (3a)}

According to the general procedure, $8 \mathbf{a}(389 \mathrm{mg}, 0.801 \mathrm{mmol})$ and Pearlman's catalyst $(129 \mathrm{mg})$ were reacted. After chromatographic purification over Silica $\mathrm{Gel} 60 \mathrm{~N}\left(\mathrm{CH}_{2} \mathrm{Cl}_{2}\right), 3 \mathbf{a}$ was obtained as colorless solid (308 mg, 97\%). Recrystallization from $\mathrm{CH}_{2} \mathrm{Cl}_{2}$-hexane gave colorless needles. $\mathrm{Mp}$ 195.5-196 ${ }^{\circ} \mathrm{C}$; IR (KBr): 3351, 1710, 1537, 1469, 1438, 1311, 1249, $1181 \mathrm{~cm}^{-1}$; ${ }^{1} \mathrm{H}$ NMR (400 MHz, $\left.\mathrm{CDCl}_{3}\right): \delta 3.77(\mathrm{~s}, 12 \mathrm{H}), 6.73-6.77(\mathrm{~m}, 4 \mathrm{H}), 7.01-7.05(\mathrm{~m}, 4 \mathrm{H}), 9.80$ (br s, $\left.1 \mathrm{H}\right) ;{ }^{13} \mathrm{C}$ NMR $(100 \mathrm{MHz}$, $\left.\mathrm{CDCl}_{3}\right): \delta 51.7,55.0,112.8,121.0,125.0,131.1,131.8,158.3,160.6$. Anal. Calcd for $\mathrm{C}_{22} \mathrm{H}_{21} \mathrm{NO}_{6}: \mathrm{C}_{\text {, }}$ 66.83; H, 5.35; N, 3.54. Found: C, 66.75; H, 5.44; N, 3.45 . 


\section{Dimethyl 3,4-diphenylpyrrole-2,5-dicarboxylate (3b)}

According to the general procedure, $8 \mathbf{b}(3.68 \mathrm{~g}, 8.64 \mathrm{mmol})$ and Pearlman's catalyst $(368 \mathrm{mg})$ were reacted. After chromatographic purification over Silica Gel $60 \mathrm{~N}\left(\mathrm{CH}_{2} \mathrm{Cl}_{2}\right), 3 \mathbf{b}$ was obtained as colorless solid (2.89 g, quant.). Recrystallization from $\mathrm{CH}_{2} \mathrm{Cl}_{2}-\mathrm{Et}_{2} \mathrm{O}$ gave colorless cube. Mp 195-196 ${ }^{\circ} \mathrm{C}$; IR (KBr): 3310, 1710, 1462, 1429, 1297, 1242, $1157 \mathrm{~cm}^{-1} ;{ }^{1} \mathrm{H}$ NMR (400 MHz, $\left.\mathrm{CDCl}_{3}\right): \delta 3.75(\mathrm{~s}, 6 \mathrm{H})$, 7.08-7.13 (m, 4H), 7.17-7.21 (m, 6H), 9.89 (br s, 1H); $\left.{ }^{13} \mathrm{C} \mathrm{NMR} \mathrm{(100} \mathrm{MHz,} \mathrm{CDCl}_{3}\right): \delta 51.7,121.1,126.8$, 127.2, 130.6, 131.4, 132.7, 160.5. Anal. Calcd for $\mathrm{C}_{20} \mathrm{H}_{17} \mathrm{NO}_{4}: \mathrm{C}, 71.63 ; \mathrm{H}, 5.11 ; \mathrm{N}, 4.18$. Found: C, $71.62 ; \mathrm{H}, 5.19 ; \mathrm{N}, 4.12$.

\section{Dimethyl 3,4-bis(4-isopropoxyphenyl)pyrrole-2,5-dicarboxylate (3c)}

According to the general procedure, 8c $(7.02 \mathrm{~g}, 13.0 \mathrm{mmol})$ and Pearlman's catalyst $(751 \mathrm{mg})$ were reacted. After chromatographic purification over Silica $\mathrm{Gel} 60 \mathrm{~N}\left(\mathrm{CH}_{2} \mathrm{Cl}_{2}\right), 3 \mathrm{c}$ was obtained as colorless solid (5.56 g, 95\%). Recrystallization from $\mathrm{CH}_{2} \mathrm{Cl}_{2}$-pentane gave colorless granules. Mp 148-149 ${ }^{\circ} \mathrm{C}$; IR (KBr): 3290, 1703, 1534, 1464, 1297, 1246, 1185, 1120, 1106, $955 \mathrm{~cm}^{-1} ;{ }^{1} \mathrm{H} \mathrm{NMR} 400 \mathrm{MHz}, \mathrm{CDCl}_{3}$ ): $\delta 1.31(\mathrm{~d}, J=6.1 \mathrm{~Hz}, 12 \mathrm{H}), 3.77(\mathrm{~s}, 6 \mathrm{H}), 4.44-4.55(\mathrm{~m}, 2 \mathrm{H}), 6.70-6.74(\mathrm{~m}, 4 \mathrm{H}), 6.98-7.02(\mathrm{~m}, 4 \mathrm{H}), 9.80$ (br s, $1 \mathrm{H}) ;{ }^{13} \mathrm{C}$ NMR $\left(100 \mathrm{MHz}, \mathrm{CDCl}_{3}\right): \delta 22.1,51.7,69.6,114.6,120.9,124.7,131.2,131.8,156.7$, 160.6. Anal. Calcd for $\mathrm{C}_{26} \mathrm{H}_{29} \mathrm{NO}_{6}$ : C, 69.16; H, 6.47; N, 3.10. Found: C, 69.16; H, 6.36; N, 3.01 .

\section{Dimethyl 3,4-bis(4-fluorophenyl)pyrrole-2,5-dicarboxylate (3d)}

According to the general procedure, 8d (462 mg, $1.00 \mathrm{mmol})$ and Pearlman's catalyst (49 $\mathrm{mg})$ were reacted. After chromatographic purification over Silica Gel $60 \mathrm{~N}$ (hexane- $\mathrm{CH}_{2} \mathrm{Cl}_{2}=1: 5$ ), 3d was obtained as colorless solid (329 mg, 89\%). Recrystallization from $\mathrm{CH}_{2} \mathrm{Cl}_{2}$-hexane gave colorless needles. $\mathrm{Mp}$ 174-175 ${ }^{\circ} \mathrm{C}$; IR (KBr): 3319, 1725, 1691, 1475, 1463, 1294, 1255, 1223, $1160 \mathrm{~cm}^{-1}$; ${ }^{1} \mathrm{H}$ NMR (400 MHz, $\left.\mathrm{CDCl}_{3}\right): \delta 3.77(\mathrm{~s}, 6 \mathrm{H}), 6.84-6.94(\mathrm{~m}, 4 \mathrm{H}), 7.03-7.09(\mathrm{~m}, 4 \mathrm{H}), 9.90$ (br s, $\left.1 \mathrm{H}\right) ;{ }^{13} \mathrm{C} \mathrm{NMR}(100 \mathrm{MHz}$, $\left.\mathrm{CDCl}_{3}\right): \delta 51.8,114.4(\mathrm{~d}, J=22 \mathrm{~Hz}), 121.2,128.4(\mathrm{~d}, J=3.3 \mathrm{~Hz}), 130.3,132.2(\mathrm{~d}, J=8.3 \mathrm{~Hz}), 160.4,161.8$ (d, $J=246 \mathrm{~Hz}$ ). Anal. Calcd for $\mathrm{C}_{20} \mathrm{H}_{15} \mathrm{~F}_{2} \mathrm{NO}_{4}: \mathrm{C}, 64.69 ; \mathrm{H}, 4.07 ; \mathrm{N}, 3.77$. Found: C, 64.58; H, 3.95; N, 3.68 .

\section{Dimethyl 3,4-bis(2-methoxyphenyl)pyrrole-2,5-dicarboxylate (3f)}

According to the general procedure, $8 \mathbf{f}(195 \mathrm{mg}, 0.402 \mathrm{mmol})$ and Pearlman's catalyst (100 $\mathrm{mg})$ were reacted. After chromatographic purification over Silica Gel $60 \mathrm{~N}\left(\mathrm{CH}_{2} \mathrm{Cl}_{2} \sim \mathrm{CH}_{2} \mathrm{Cl}_{2}\right.$-EtOAc=10:1), 3f was obtained as colorless solid (140 mg, 88\%). Recrystallization from $\mathrm{CH}_{2} \mathrm{Cl}_{2}$-pentane gave colorless granules. Mp 203-205 ${ }^{\circ} \mathrm{C}$; IR (KBr): 3290, 1713, 1694, 1470, 1434, 1300, 1270, 1248, $1157 \mathrm{~cm}^{-1} ;{ }^{1} \mathrm{H}$ NMR (400 MHz, $\mathrm{CDCl}_{3}$ ): $\delta 3.53$ (br s, 6H), $3.72(\mathrm{~s}, 6 \mathrm{H}), 6.76(\mathrm{dd}, J=1.0$ and $7.5 \mathrm{~Hz}, 2 \mathrm{H}), 6.78(\mathrm{dt}, J=$ 
1.0 and $7.5 \mathrm{~Hz}, 2 \mathrm{H}), 6.98(\mathrm{dd}, J=1.8$ and $7.5 \mathrm{~Hz}, 2 \mathrm{H}), 7.16(\mathrm{dt}, J=1.8$ and $7.5 \mathrm{~Hz}, 2 \mathrm{H}), 9.86$ (br s, $1 \mathrm{H}$ ); ${ }^{13} \mathrm{C}$ NMR (100 MHz, $\left.\mathrm{CDCl}_{3}\right): \delta 51.5,55.1,110.2,119.5,122.0,122.7,127.8,128.4,131.6,157.0,160.6$. Anal. Calcd for $\mathrm{C}_{22} \mathrm{H}_{21} \mathrm{NO}_{6}$ : C, 66.83; H, 5.35; N, 3.54. Found: C, 66.76; H, 5.41; N, 3.37.

\section{Dimethyl 3,4-bis(3,4-dimethoxyphenyl)pyrrole-2,5-dicarboxylate (3g)}

According to the general procedure, $8 \mathrm{~g}(546 \mathrm{mg}, 1.00 \mathrm{mmol})$ and Pearlman's catalyst $(55 \mathrm{mg})$ were reacted. After chromatographic purification over Silica Gel $60 \mathrm{~N}\left(\mathrm{CH}_{2} \mathrm{Cl}_{2}\right), 3 \mathrm{~g}$ was obtained as colorless solid (432 mg, 95\%). Recrystallization from $\mathrm{CH}_{2} \mathrm{Cl}_{2}$-hexane gave colorless needles. $\mathrm{Mp}$ 168.5-169.5 ${ }^{\circ} \mathrm{C}$; IR (KBr): 3313, 1719, 1683, 1531, 1469, 1438, 1319, 1279, 1245, 1138, $1024 \mathrm{~cm}^{-1} ;{ }^{1} \mathrm{H}$ NMR (400 MHz, $\left.\mathrm{CDCl}_{3}\right): \delta 3.64(\mathrm{~s}, 6 \mathrm{H}), 3.79(\mathrm{~s}, 6 \mathrm{H}), 3.85(\mathrm{~s}, 6 \mathrm{H}), 6.61-6.63(\mathrm{~m}, 2 \mathrm{H}), 6.71-6.77(\mathrm{~m}, 4 \mathrm{H})$, 9.83 (br s, $1 \mathrm{H}) ;{ }^{13} \mathrm{C} \mathrm{NMR}\left(100 \mathrm{MHz}, \mathrm{CDCl}_{3}\right.$ ): $\delta 51.8,55.7,55.7,110.2,114.2,120.9,123.2,125.2,131.1$, 147.7, 147.9, 160.6. Anal. Calcd for $\mathrm{C}_{24} \mathrm{H}_{25} \mathrm{NO}_{8}: \mathrm{C}, 63.29 ; \mathrm{H}, 5.53 ; \mathrm{N}, 3.08$. Found: C, 63.33; H, 5.47; N, 2.98 .

\section{Dimethyl 3,4-bis(3,4,5-trimethoxyphenyl)pyrrole-2,5-dicarboxylate (3h)}

According to the general procedure, $\mathbf{8 h}(612 \mathrm{mg}, 1.01 \mathrm{mmol})$ and Pearlman's catalyst $(67 \mathrm{mg})$ were reacted. After chromatographic purification over Silica Gel $60 \mathrm{~N}\left(\mathrm{CH}_{2} \mathrm{Cl}_{2}\right), 3 \mathbf{h}$ was obtained as colorless solid (502 mg, 96\%). Recrystallization from $\mathrm{CH}_{2} \mathrm{Cl}_{2}$-hexane gave colorless needles. Mp 166-167 ${ }^{\circ} \mathrm{C}$; IR (KBr): 3273, 1730, 1702, 1586, 1480, 1460, 1412, 1344, 1239, $1124 \mathrm{~cm}^{-1}$; ${ }^{1} \mathrm{H}$ NMR (400 MHz, $\left.\mathrm{CDCl}_{3}\right): \delta 3.65(\mathrm{~s}, 12 \mathrm{H}), 3.83(\mathrm{~s}, 12 \mathrm{H}), 6.38(\mathrm{~s}, 4 \mathrm{H}), 9.90($ br s, $1 \mathrm{H}) ;{ }^{13} \mathrm{C} \mathrm{NMR}\left(100 \mathrm{MHz}, \mathrm{CDCl}_{3}\right): \delta 51.9$, 56.0, 60.8, 108.4, 120.9, 128.0, 131.0, 137.1, 152.2, 160.4. Anal. Calcd for $\mathrm{C}_{26} \mathrm{H}_{29} \mathrm{NO}_{10}$ : C, 60.58; H, 5.67; N, 2.72. Found: C, 60.57; H, 5.68; N, 2.60.

\section{Dimethyl 3,4-bis(4,5-dimethoxy-2-methoxymethoxyphenyl)pyrrole-2,5-dicarboxylate (3i)}

According to the general procedure, $8 \mathbf{i}(1.22 \mathrm{~g}, 1.83 \mathrm{mmol})$ and Pearlman's catalyst $(128 \mathrm{mg})$ were reacted. After chromatographic purification over Silica Gel 60N (hexane-EtOAc=1:2), 3i was obtained as colorless solid (993 mg, 94\%). Recrystallization from $\mathrm{CH}_{2} \mathrm{Cl}_{2}-\mathrm{Et}_{2} \mathrm{O}$ gave colorless granules. $\mathrm{Mp}$ 150.5-151.5 ${ }^{\circ} \mathrm{C}$; IR (KBr): $3277,1713,1494,1233,1152,1099,1011 \mathrm{~cm}^{-1} ;{ }^{1} \mathrm{H} \mathrm{NMR}$ (400 MHz, $\mathrm{CDCl}_{3}$ ): $\delta 3.31$ (br s, 6H), 3.58 (br s, 6H), 3.77 (s, 6H), 3.84 (s, 6H), 4.61 (br s, 1H), 4.85 (br s, 2H), 5.02 (br s, 1H), 6.50 (br s, 2H), 6.77 (br s, 2H), 9.84 (br s, 1H); ${ }^{13} \mathrm{C} \mathrm{NMR} \mathrm{(100} \mathrm{MHz,} \mathrm{CDCl}_{3}$ ): $\delta$ 51.7, 55.7, 55.9, 56.1, 96.8, 101.4, 114.5, 122.2, 127.6, 143.6, 149.0, 150.0, 160.8. Anal. Calcd for $\mathrm{C}_{28} \mathrm{H}_{33} \mathrm{NO}_{12}$ : C, 58.43; H, 5.78; N, 2.43. Found: C, 58.48; H, 5.82; N, 2.26. 
Under an argon atmosphere, a mixture of $8 \mathbf{e}(509 \mathrm{mg}, 1.03 \mathrm{mmol})$, anisole (140 $\mu \mathrm{L}, 1.29 \mathrm{mmol})$, and $98 \%$ $\mathrm{H}_{2} \mathrm{SO}_{4}(36 \mu \mathrm{L}, 0.68 \mathrm{mmol})$ in trifluoroacetic acid $(1.2 \mathrm{~mL})$ was refluxed for $0.5 \mathrm{~h}$. After being cooled to $\mathrm{rt}$, the mixture was evaporated under reduced pressure. The residue was basified with saturated aqueous $\mathrm{NaHCO}_{3}$ and the mixture was extracted with $\mathrm{CH}_{2} \mathrm{Cl}_{2}$. The extract was washed successively with water and brine, dried over $\mathrm{Na}_{2} \mathrm{SO}_{4}$, and evaporated under reduced pressure. The residue was purified by column chromatography over Silica Gel $60 \mathrm{~N}$ (hexane- $\mathrm{CH}_{2} \mathrm{Cl}_{2}=1: 5 \sim \mathrm{CH}_{2} \mathrm{Cl}_{2}$ ) to give $\mathbf{3 e}$ as colorless solid (333 mg, 80\%). Recrystallization from $\mathrm{Et}_{2} \mathrm{O}$-pentane gave colorless granules. Mp 191.5-193 ${ }^{\circ} \mathrm{C}$; IR (KBr): 3281, 1713, 1462, 1436, 1298, 1248, 1163, 1091, $1027 \mathrm{~cm}^{-1} ;{ }^{1} \mathrm{H}$ NMR (400 MHz, $\left.\mathrm{CDCl}_{3}\right): \delta 3.77$ $(\mathrm{s}, 6 \mathrm{H}), 7.00-7.04(\mathrm{~m}, 4 \mathrm{H}), 7.17-7.21(\mathrm{~m}, 4 \mathrm{H}), 9.92$ (br s, $1 \mathrm{H}) ;{ }^{13} \mathrm{C} \mathrm{NMR}\left(100 \mathrm{MHz}, \mathrm{CDCl}_{3}\right): \delta 51.9$, 121.3, 127.7, 129.9, 130.9, 131.9, 133.1, 160.2. HREIMS m/z. Calcd for $\mathrm{C}_{20} \mathrm{H}_{15} \mathrm{Cl}_{2} \mathrm{NO}_{4}\left(\mathrm{M}^{+}\right)$: 403.0378 . Found: 403.0379.

\section{Dimethyl}

$N$-benzyl-3-(3,4-dimethoxyphenyl)-4-(trifluoromethanesulfonyloxy)pyrrole-2,5-

\section{dicarboxylate (9)}

Under an argon atmosphere, a degassed solution of $\mathrm{Na}_{2} \mathrm{CO}_{3}(4.23 \mathrm{~g}, 39.9 \mathrm{mmol})$ in water $(12 \mathrm{~mL})$ was added to a solution of $7(3.46 \mathrm{~g}, 6.08 \mathrm{mmol}), 2 \mathrm{~g}(1.10 \mathrm{~g}, 6.05 \mathrm{mmol})$ and $\mathrm{Pd}\left(\mathrm{PPh}_{3}\right)_{4}(140 \mathrm{mg}, 0.121$ $\mathrm{mmol})$ in THF $(120 \mathrm{~mL})$ at $\mathrm{rt}$ and the mixture was refluxed for $4 \mathrm{~h}$. The mixture was cooled to $\mathrm{rt}$ and evaporated under reduced pressure. The products were extracted with $\mathrm{CH}_{2} \mathrm{Cl}_{2}$ and the extract was washed successively with water and brine, dried over $\mathrm{Na}_{2} \mathrm{SO}_{4}$, and evaporated under reduced pressure. The residue was purified successively by column chromatography over Silica Gel 60N (toluene toluene-EtOAc=5:1) and over Silica Gel $60 \mathrm{~N}\left(\mathrm{CH}_{2} \mathrm{Cl}_{2}\right)$ to give 9 as colorless solid $(2.54 \mathrm{~g}$, 75\%), 3,4-diarylated $8 \mathrm{~g}$ (258 mg, 8\%), and unreacted 7 (461 mg, 13\%). Recrystallization from $\mathrm{CH}_{2} \mathrm{Cl}_{2}$-hexane gave colorless granules. $\quad$ Mp 131-132 ${ }^{\circ} \mathrm{C}$; IR (KBr): 1728, 1442, 1425, 1294, 1265, 1212, $1139 \mathrm{~cm}^{-1} ;{ }^{1} \mathrm{H}$ NMR (400 MHz, $\left.\mathrm{CDCl}_{3}\right): \delta 3.58(\mathrm{~s}, 3 \mathrm{H}), 3.86(\mathrm{~s}, 3 \mathrm{H}), 3.88(\mathrm{~s}, 3 \mathrm{H}), 3.92(\mathrm{~s}, 3 \mathrm{H}), 6.09(\mathrm{~s}, 2 \mathrm{H}), 6.82-6.90$ $(\mathrm{m}, 3 \mathrm{H}), 7.01-7.05(\mathrm{~m}, 2 \mathrm{H}), 7.21-7.27(\mathrm{~m}, 1 \mathrm{H}), 7.27-7.33(\mathrm{~m}, 2 \mathrm{H}) ;{ }^{13} \mathrm{C} \mathrm{NMR}\left(100 \mathrm{MHz}, \mathrm{CDCl}_{3}\right)$ : 49.6, 51.9, 51.9, 55.8, 55.9, 110.5, 113.2, $117.8,117.9$ (q, $J=320 \mathrm{~Hz}), 121.9,122.7,123.2,123.2,126.1,127.3$, 128.5, 136.0, 137.3, 148.3, 148.8, 159.1, 160.8. Anal. Calcd for $\mathrm{C}_{24} \mathrm{H}_{22} \mathrm{~F}_{3} \mathrm{NO}_{9} \mathrm{~S}: \mathrm{C}, 51.71 ; \mathrm{H}, 3.98 ; \mathrm{N}$, 2.51. Found: C, 51.90; H, 3.93; N, 2.40 .

\section{Dimethyl $N$-benzyl-3-(3,4-dimethoxyphenyl)-4-(4-methoxyphenyl)pyrrole-2,5-dicarboxylate (10)}

Under an argon atmosphere, a degassed solution of $\mathrm{Na}_{2} \mathrm{CO}_{3}(2.10 \mathrm{~g}, 19.8 \mathrm{mmol})$ in water $(6.0 \mathrm{~mL})$ was added to a solution of 9 (1.67 g, $3.00 \mathrm{mmol})$, 2a $(685 \mathrm{mg}, 4.51 \mathrm{mmol})$ and $\mathrm{Pd}\left(\mathrm{PPh}_{3}\right)_{4}(145 \mathrm{mg}, 0.125$ $\mathrm{mmol})$ in THF $(60 \mathrm{~mL})$ at $\mathrm{rt}$ and the mixture was refluxed for $20 \mathrm{~h}$. The mixture was cooled to $\mathrm{rt}$ and evaporated under reduced pressure. The products were extracted with $\mathrm{CH}_{2} \mathrm{Cl}_{2}$ and the extract was 
washed successively with water and brine, dried over $\mathrm{Na}_{2} \mathrm{SO}_{4}$, and evaporated under reduced pressure. The residue was purified successively by column chromatography over Silica Gel 60N (toluene-EtOAc=10:1) and over Chromatorex NH-DM1020 silica gel (EtOAc) to give $\mathbf{1 0}$ as colorless

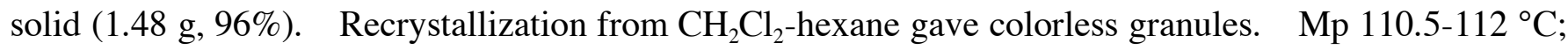
IR (KBr): 1715, 1535, 1435, 1299, 1245, 1202, 1173, $1025 \mathrm{~cm}^{-1} ;{ }^{1} \mathrm{H}$ NMR (400 MHz, $\left.\mathrm{CDCl}_{3}\right): \delta 3.53$ (s, $3 \mathrm{H}), 3.55(\mathrm{~s}, 3 \mathrm{H}), 3.62(\mathrm{~s}, 3 \mathrm{H}), 3.75(\mathrm{~s}, 3 \mathrm{H}), 3.83(\mathrm{~s}, 3 \mathrm{H}), 6.01(\mathrm{~s}, 2 \mathrm{H}), 6.51(\mathrm{~d}, J=2.0 \mathrm{~Hz}, 1 \mathrm{H}), 6.62(\mathrm{dd}$, $J=2.0$ and $8.3 \mathrm{~Hz}, 1 \mathrm{H}), 6.70(\mathrm{~d}, J=8.3 \mathrm{~Hz}, 1 \mathrm{H}), 6.70-6.75(\mathrm{~m}, 2 \mathrm{H}), 6.94-6.98(\mathrm{~m}, 2 \mathrm{H}), 7.09-7.13(\mathrm{~m}, 2 \mathrm{H})$, 7.19-7.25 (m, 1H), 7.27-7.32 (m, 2H); $\left.{ }^{13} \mathrm{C} \mathrm{NMR} \mathrm{(100} \mathrm{MHz,} \mathrm{CDCl}_{3}\right)$ : 49.6, 51.4, 51.5, 55.0, 55.6, 55.6, $110.0,112.7,113.8,122.7,124.2,124.4,126.2$, 126.5, 126.9, 128.3, 130.6, 130.8, 131.3, 138.5, 147.4, 147.6, 158.1, 161.9, 162.1. Anal. Calcd for $\mathrm{C}_{30} \mathrm{H}_{29} \mathrm{NO}_{7}: \mathrm{C}, 69.89 ; \mathrm{H}, 5.67 ; \mathrm{N}, 2.72$. Found: C, 69.73; H, $5.64 ; \mathrm{N}, 2.63$.

\section{Dimethyl 3-(3,4-dimethoxyphenyl)-4-(4-methoxyphenyl)pyrrole-2,5-dicarboxylate (11)}

Under an argon atmosphere, ammonium formate $(1.93 \mathrm{~g}, 30.6 \mathrm{mmol})$ was added portionwise to a mixture of $10(523 \mathrm{mg}, 1.01 \mathrm{mmol})$, Pearlman's catalyst $(51 \mathrm{mg})$, and EtOH $(20 \mathrm{~mL})$ at rt. After being refluxed for $3 \mathrm{~h}$, the mixture was cooled to $\mathrm{rt}$ and passed through a pad of Celite. The filtrate was evaporated under reduced pressure. The residue was purified by column chromatography over Silica Gel 60N $\left(\mathrm{CH}_{2} \mathrm{Cl}_{2}\right)$ to give 11 as colorless solid $(298 \mathrm{mg}, 69 \%)$. Recrystallization from $\mathrm{CH}_{2} \mathrm{Cl}_{2}$-pentane gave

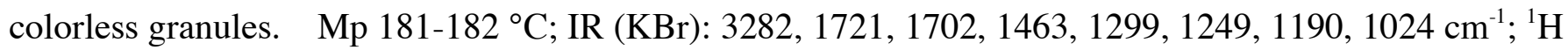
NMR (400 MHz, $\left.\mathrm{CDCl}_{3}\right): \delta 3.61(\mathrm{~s}, 3 \mathrm{H}), 3.77$ (s, 3H), 3.77 (s, 3H), 3.79 (s, 3H), 3.85 (s, 3H), 6.56-6.58 $(\mathrm{m}, 1 \mathrm{H}), 6.72-6.79(\mathrm{~m}, 4 \mathrm{H}), 7.01-7.06(\mathrm{~m}, 2 \mathrm{H}), 9.82$ (br s, $1 \mathrm{H}) ;{ }^{13} \mathrm{C} \mathrm{NMR}\left(100 \mathrm{MHz}, \mathrm{CDCl}_{3}\right): 51.7,55.1$, 55.6, 55.6, 110.0, 112.8, 114.2, 120.9, 121.0, 123.3, 125.1, 125.1, 131.1, 131.1, 131.7, 147.6, 147.7, 158.4, 160.6. HREIMS $m / z$. Calcd for $\mathrm{C}_{23} \mathrm{H}_{23} \mathrm{NO}_{7}\left(\mathrm{M}^{+}\right)$: 425.1475. Found: 425.1469 .

\section{3,4-Bis(4-isopropoxyphenyl)-5-methoxycarbonylpyrrole-2-carboxylic acid (12)}

Under an argon atmosphere, 3c (2.71 g, $6.00 \mathrm{mmol})$ and $\mathrm{LiOH} \cdot \mathrm{H}_{2} \mathrm{O}(126 \mathrm{mg}, 3.00 \mathrm{mmol})$ were dissolved in a degassed mixture of THF (36 mL), MeOH $(12 \mathrm{~mL})$, and water $(12 \mathrm{~mL})$. After being stirred for $44 \mathrm{~h}$ at $\mathrm{rt}$, the mixture was acidified with $3 \mathrm{M}$ aqueous $\mathrm{HCl}$ to $\mathrm{pH} 1$ and evaporated under reduced pressure. The product was extracted with EtOAc and the extract was washed with brine, dried over $\mathrm{Na}_{2} \mathrm{SO}_{4}$, and evaporated under reduced pressure. The residue was purified by column chromatography over Silica Gel 60N [hexane-EtOAc=1:1 EtOAc-water (upper phase)] to give 12 as colorless solid (1.23 g, 47\%) and unreacted 3c (1.38 g, 51\%). Recrystallization from $\mathrm{CH}_{2} \mathrm{Cl}_{2}$-pentane gave colorless powder. Mp 219-220 ${ }^{\circ} \mathrm{C}$; IR (KBr): 3308, 1700, 1466, 1298, 1242, 1119, $952 \mathrm{~cm}^{-1} ;{ }^{1} \mathrm{H}$ NMR (400 MHz, DMSO- $\left.d_{6}\right): \delta$ $1.22(\mathrm{~d}, J=6.0 \mathrm{~Hz}, 12 \mathrm{H}), 3.64$ (s, 3H), 4.47-4.58 (m, 2H), 6.67-6.72 (m, 4H), 6.89-6.95 (m, 4H), 11.94 (br 
s, $1 \mathrm{H}) ;{ }^{13} \mathrm{C}$ NMR (100 MHz, DMSO- $\left.d_{6}\right): \delta 21.7,21.8,51.1,68.7,68.7,113.9,114.0,120.6,122.5,125.1$, 125.3, 129.4, 130.0, 131.5, 131.6, 155.7, 155.8, 160.1, 161.2. Anal. Calcd for $\mathrm{C}_{25} \mathrm{H}_{27} \mathrm{NO}_{6}: \mathrm{C}, 68.63 ; \mathrm{H}$, 6.22; N, 3.20. Found: C, 68.63; H, 6.11; N, 3.14.

\section{Methyl 3,4-bis(4-isopropoxyphenyl)pyrrole-2-carboxylate (13)}

Under an argon atmosphere, a mixture of 12 (500 mg, $1.14 \mathrm{mmol}$ ) and copper(I) oxide (164 mg, 1.14 $\mathrm{mmol})$ in quinoline $(10 \mathrm{~mL})$ was heated at $220^{\circ} \mathrm{C}$ for $7 \mathrm{~min}$. The mixture was cooled to $\mathrm{rt}$ and passed through a pad of Celite. The filtrate was diluted with $\mathrm{CH}_{2} \mathrm{Cl}_{2}$, washed successively with $6 \mathrm{M}$ aqueous $\mathrm{HCl}$, water and brine, dried over $\mathrm{Na}_{2} \mathrm{SO}_{4}$, and evaporated under reduced pressure. The residue was purified by flash chromatography over Silica Gel 60N (hexane-EtOAc=3:1) to give $\mathbf{1 3}$ as yellow solid (361 mg, 80\%). Recrystallization from EtOAc-hexane gave yellow needles. The spectroscopic data are identical with those reported in ref. 26.

\section{ACKNOWLEDGEMENTS}

The authors acknowledge the Ministry of Education, Culture, Sports, Science and Technology of Japan for financial support; Grant-in-Aid for Scientific Research (B) (No. 20310135).

\section{REFERENCES AND NOTES}

1. For recent reviews, see: (a) J. Kluza, P. Marchetti, and C. Bailly, 'Modern Alkaloids: Structure, Isolation, Synthesis and Biology,' ed. by E. Fattorusso and O. Taglialatela-Scafati, Wiley-VCH, Weinheim, 2008, pp. 171-187. (b) H. Fan, J. Peng, M. T. Hamann, and J.-F. Hu, Chem. Rev., 2008, 108, 264.

2. W. Y. Yoshida, K. K. Lee, A. R. Carroll, and P. J. Scheuer, Helv. Chim. Acta, 1992, 75, 1721.

3. H. Kang and W. Fenical, J. Org. Chem., 1997, 62, 3254.

4. J. A. Palermo, M. F. R. Brasco, and A. M. Seldes, Tetrahedron, 1996, 52, 2727.

5. (a) A. Rudi, I. Goldberg, Z. Stein, F. Frolow, Y. Benayahu, M. Schleyer, and Y. Kashman, J. Org. Chem., 1994, 59, 999. (b) S. Loya, A. Rudi, Y. Kashman, and A. Hizi, Biochem. J., 1999, 344, 85.

(c) A. Rudi, T. Evan, M. Aknin, and Y. Kashman, J. Nat. Prod., 2000, 63, 832.

6. G. W. Chan, T. Francis, D. R. Thureen, P. H. Offen, N. J. Pierce, J. W. Westley, R. K. Johnson, and D. J. Faulkner, J. Org. Chem., 1993, 58, 2544.

7. Y. Kashman, G. Koren-Goldshlager, M. D. G. Gravalos, and M. Schleyer, Tetrahedron Lett., 1999, 40, 997.

8. K. Warabi, S. Matsunaga, R. W. M. van Soest, and N. Fusetani, J. Org. Chem., 2003, 68, 2765.

9. For reviews, see: (a) P. Cironi, F. Albericio, and M. Álvarez, Progress in Heterocyclic Chemistry, 
2004, 16, 1. (b) S. T. Handy and Y. Zhang, Org. Prep. Proc. Int., 2005, 37, 411. (c) F. Bellina and R. Rossi, Tetrahedron, 2006, 62, 7213.

10. A. Fürstner, H. Weintritt, and A. Hupperts, J. Org. Chem., 1995, 60, 6637.

11. (a) A. Heim, A. Terpin, and W. Steglich, Angew. Chem., Int. Ed. Engl., 1997, 36, 155. (b) H. Ebel, A. Terpin, and W. Steglich, Tetrahedron Lett., 1998, 39, 9165. (c) C. Peschko and W. Steglich, Tetrahedron Lett., 2000, 41, 9477. (d) C. Peschko, C. Winklhofer, and W. Steglich, Chem. Eur. J., 2000, 6, 1147. (e) C. Peschko, C. Winklhofer, A. Terpin, and W. Steglich, Synthesis, 2006, 3048. (f) C. Hinze, A. Kreipl, A. Terpin, and W. Steglich, Synthesis, 2007, 608.

12. (a) F. Ishibashi, Y. Miyazaki, and M. Iwao, Tetrahedron, 1997, 53, 5951. (b) S. Ruchirawat and T. Mutarapat, Tetrahedron Lett., 2001, 42, 1205. (c) P. Ploypradith, W. Jinaglueng, C. Pavaro, and S. Ruchirawat, Tetrahedron Lett., 2003, 44, 1363. (d) P. Ploypradith, C. Mahidol, P. Sahakitpichan, S. Wongbundit, and S. Ruchirawat, Angew. Chem. Int. Ed., 2004, 43, 866.

13. (a) M. Banwell, B. Flynn, and D. Hockless, Chem. Commun., 1997, 2259. (b) C. P. Ridley, M. V. R. Reddy, G. Rocha, F. D. Bushman, and D. J. Faulkner, Bioorg. Med. Chem., 2002, 10, 3285. (c) P. Cironi, I. Manzanares, F. Albericio, and M. Álvarez, Org. Lett., 2003, 5, 2959. (d) P. Cironi, C. Cuevas, F. Albericio, and M. Álvarez, Tetrahedron, 2004, 60, 8669. (e) M. Díaz, E. Guitián, and L. Castedo, Synlett, 2001, 1164.

14. J. L. Bullington, R. R. Wolff, and P. F. Jackson, J. Org. Chem., 2002, 67, 9439.

15. (a) D. L. Boger, C. W. Boyce, M. A. Labroli, C. A. Sehon, and Q. Jin, J. Am. Chem. Soc., 1999, 121, 54. (b) D. L. Boger, D. R. Soenen, C. W. Boyce, M. P. Hedrick, and Q. Jin, J. Org. Chem., 2000, 65 , 2479. (c) A. Hamasaki, J. M. Zimpleman, I. Hwang, and D. L. Boger, J. Am. Chem. Soc., 2005, 127, 10767.

16. (a) J. T. Gupton, K. E. Krumpe, B. S. Burnham, T. M. Webb, J. S. Shuford, and J. A. Sikorski, Tetrahedron, 1999, 55, 14515. (b) J. T. Gupton, S. C. Clough, R. B. Miller, J. R. Lukens, C. A. Henry, R. P. F. Kanters, and J. A. Sikorski, Tetrahedron, 2003, 59, 207. (c) J. T. Gupton, R. B. Miller, K. E. Krumpe, S. C. Clough, E. J. Banner, R. P. F. Kanters, K. X. Du, K. M. Keertikar, N. E. Lauerman, J. M. Solano, B. R. Adams, D. W. Callahan, B. A. Little, A. B. Scharf, and J. A. Sikorski, Tetrahedron, 2005, 61, 1845.

17. S. Kim, S. Son, and H. Kang, Bull. Korean Chem. Soc., 2001, 22, 1403.

18. A. Namsa-aid and S. Ruchirawat, Org. Lett., 2002, 4, 2633.

19. (a) M. G. Banwell, B. L. Flynn, E. Hamel, and D. C. R. Hockless, Chem. Commun., 1997, 207. (b) M. G. Banwell, B. L. Flynn, D. C. R. Hockless, R. W. Longmore, and A. D. Rae, Aust. J. Chem., 1999, 52, 755. (c) J.-H. Liu, Q.-C. Yang, T. C. W. Mak, and H. N. C. Wong, J. Org. Chem., 2000, 65, 3587. (d) A. Fürstner, H. Krause, and O. R. Thiel, Tetrahedron, 2002, 58, 6373. (e) M. G. 
Banwell, A. M. Bray, A. J. Edwards, and D. J. Wong, J. Chem. Soc., Perkin Trans. 1, 2002, 1340. (f) S. T. Handy, H. Bregman, J. Lewis, X. Zhang, and Y. Zhang, Tetrahedron Lett., 2003, 44, 427. (g) M. R. Heinrich, W. Steglich, M. G. Banwell, and Y. Kashman, Tetrahedron, 2003, 59, 9239. (h) S. T. Handy, Y. Zhang, and H. Bregman, J. Org. Chem., 2004, 69, 2362. (i) C. A. Olsen, N. Parera, F. Albericio, and M. Álvarez, Tetrahedron Lett., 2005, 46, 2041. (j) J. A. Smith, S. Ng, and J. White, Org. Biomol. Chem., 2006, 4, 2477. (k) M. G. Banwell, T. E. Goodwin, S. Ng, J. A. Smith, and D. J. Wong, Eur. J. Org. Chem., 2006, 3043.

20. M. Iwao, T. Takeuchi, N. Fujikawa, T. Fukuda, and F. Ishibashi, Tetrahedron Lett., 2003, 44, 4443.

21. (a) N. Fujikawa, T. Ohta, T. Yamaguchi, T. Fukuda, F. Ishibashi, and M. Iwao, Tetrahedron, 2006, 62, 594. (b) T. Yamaguchi, T. Fukuda, F. Ishibashi, and M. Iwao, Tetrahedron Lett., 2006, 47, 3755. 22. S. F. Vasilevsky, S. V. Klyatskaya, E. V. Tretyakov, and J. Elguero, Heterocycles, 2003, $60,879$.

23. A. Merz, R. Schropp, and J. Lex, Angew. Chem., Int. Ed. Engl., 1993, 32, 291.

24. W. M. Pearlman, Tetrahedron Lett., 1967, 8, 1663.

25. A. Merz, R. Schropp, and E. Dötterl, Synthesis, 1995, 795.

26. T. Fukuda, E. Sudo, K. Shimokawa, and M. Iwao, Tetrahedron, 2008, 64, 328. 